Appl. Set-Valued Anal. Optim. 2 (2020), No. 1, pp. 63-93

Available online at http://asvao.biemdas.com

https://doi.org/10.23952/asvao.2.2020.1.05

\title{
TURNPIKE PROPERTIES FOR THE ROBINSON-SOLOW-SRINIVASAN MODEL: A SURVEY OF RECENT RESULTS
}

\author{
ALEXANDER J. ZASLAVSKI \\ Department of Mathematics, The Technion - Israel Institute of Technology, 32000 Haifa, Israel
}

\begin{abstract}
In this work we survey recent results on the structure of approximate optimal programs for the Robinson-Solow-Srinivasan model. We discuss turnpike properties of approximate solutions as well as the existence of solutions of the corresponding infinite horizon problems.
\end{abstract}

Keywords. Good program; Infinite horizon problem; Overtaking optimal program; Turnpike.

\section{INTRODUCTION}

The study of the existence and the structure of solutions of optimal control problems defined on infinite intervals and on sufficiently large intervals has recently been a rapidly growing area of research; see, e.g., [1, 3, 4, 5, 6, 7, 8, 9, 10, 11, 12, 18, 34, 36, 38, 41, 42, 44, 50, 77, 79] and the references mentioned therein. These problems arise in engineering [61], in models of economic growth $[9,13,19,30,31,32,33,37,40,47,51,58,59,60,61,65,67,71,75$, $76,81]$, in the game theory $[46,78]$, in optimal control with PDE $[14,45,57,80]$ in infinite discrete models of solid-state physics related to dislocations in one-dimensional crystals [2] and in the theory of thermodynamical equilibrium for materials [35, 39]. In this paper we survey recent results on the structure of approximate solutions of optimal control problems arising in mathematical economics, related to the Robinson-Solow-Srinivasan model (RSS model), which was introduced in the sixties by Robinson, Solow and Srinivasan [48, 52, 53] and was studied by Robinson, Okishio and Stiglitz [43, 49, 54, 55, 56]. Recently, the Robinson-SolowSrinivasan model was studied by Khan and Mitra [19, 20, 21, 22, 23, 24, 25, 26], Khan and Piazza [27, 28, 29], Khan and Zaslavski [30, 31, 32, 33] and Zaslavski [62, 64, 65, 66, 68, 69, 70, 71, 72, 73, 74, 75]. As usual, for the Robinson-Solow-Srinivasan model the existence of optimal solutions over infinite horizon and the structure of solutions on finite intervals are under consideration. We discuss turnpike properties of approximate solutions as well as the existence of solutions of the corresponding infinite horizon problems.

Our paper has the following structure. In Section 2, we present the description of the Robinson-Solow-Srinivasan model and consider its basic properties. In particular, we discuss the existence of weakly optimal programs and good programs, and an average turnpike property. Infinite horizon optimal control problems, related to the Robinson-Solow-Srinivasan model, are considered in Section 3, where we discuss a convergence of good programs to the golden-rule

E-mail address: ajzasl@technion.ac.il.

Received December 17, 2019; Accepted February 8, 2020.

(C)2020 Applied Set-Valued Analysis and Optimization 
stock, the existence of overtaking optimal programs and their convergence to the golden-rule stock, and some properties of good programs. Turnpike properties for the Robinson-SolowSrinivasan model are discussed in Section 4. To have these properties means that the approximate solutions of the problems are essentially independent of the choice of an interval and endpoint conditions. It is shown that these turnpike properties hold and that they are stable under perturbations of an objective function. In Section 5, we study infinite horizon optimal control problems related to the Robinson-Solow-Srinivasan model with a nonconcave utility function. In particular, we show the existence of good programs and optimal programs using different optimality criterions. In Section 6, we consider infinite horizon optimal control problems with noautonomous optimality criterions. The utility functions, which determine the optimality criterion, are nonconcave. The class of models contains, as a particular case, the Robinson-Solow-Srinivasan model. We show the existence of good programs and optimal programs. Section 7 contains turnpike results for a class of discrete-time optimal control problems. These control problems arise in economic dynamics and describe the one-dimensional nonstationary Robinson-Solow-Srinivasan model.

\section{THE ROBINSON-SOLOW-SRINIVASAN MODEL}

In this paper we use the following notation.

Let $R^{1}\left(R_{+}^{1}\right)$ be the set of real (non-negative) numbers and let $R^{n}$ be the $n$-dimensional Euclidean space with the non-negative orthant

$$
R_{+}^{n}=\left\{x=\left(x_{1}, \ldots, x_{n}\right) \in R^{n}: x_{i} \geq 0, i=1, \ldots, n\right\} .
$$

For every pair of vectors $x=\left(x_{1}, \ldots, x_{n}\right), y=\left(y_{1}, \ldots, y_{n}\right) \in R^{n}$, define their inner product by

$$
x y=\sum_{i=1}^{n} x_{i} y_{i}
$$

and let $x>>y, x>y, x \geq y$ have their usual meaning. Namely, for a given pair of vectors $x=\left(x_{1}, \ldots, x_{n}\right), y=\left(y_{1}, \ldots, y_{n}\right) \in R^{n}$, we say that $x \geq y$, if $x_{i} \geq y_{i}$ for all $i=1, \ldots, n, x>y$ if $x \geq y$ and $x \neq y$, and $x>>y$ if $x_{i}>y_{i}$ for all $i=1, \ldots, n$.

Let $e(i), i=1, \ldots, n$, be the $i$ th unit vector in $R^{n}$, and $e$ be an element of $R_{+}^{n}$ all of whose coordinates are unity. For every $x \in R^{n}$, denote by $\|x\|$ its Euclidean norm in $R^{n}$.

Let $a=\left(a_{1}, \ldots, a_{n}\right)>>0, b=\left(b_{1}, \ldots, b_{n}\right)>>0, d \in(0,1), c_{i}=b_{i} /\left(1+d a_{i}\right), i=1, \ldots, n$.

These parameters define an economy capable of producing a finite number $n$ of alternative types of machines. For every $i=1, \ldots, n$, one unit of machine of type $i$ requires $a_{i}>0$ units of labor to construct it, and together with one unit of labor, each unit of it can produce $b_{i}>0$ units of a single consumption good. Thus, the production possibilities of the economy are represented by an (labor) input-coefficients vector, $a=\left(a_{1}, \ldots, a_{n}\right)>>0$ and an output-coefficients vector, $b=\left(b_{1}, \ldots, b_{n}\right)>>0$. Without loss of generality we assume that the types of machines are numbered such that $b_{1} \geq b_{2} \cdots \geq b_{n}$.

We assume that all machines depreciate at a rate $d \in(0,1)$. Thus the effective labor cost of producing a unit of output on a machine of type $i$ is given by $\left(1+d a_{i}\right) / b_{i}$ : the direct labor cost of producing unit output, and the indirect cost of replacing the depreciation of the machine in this production. We consider the reciprocal of the effective labor cost, the effective output that takes the depreciation into account, and denote it by $c_{i}$ for the machine of type $i$. 
In this section we assume that there is a unique machine type $\sigma$ at which effective labor cost $\left(1+d a_{i}\right) / b_{i}$ is minimal, or at which the effective output per man $b_{i} /\left(1+d a_{i}\right)$ is maximal. Thus assume the following:

there exists $\sigma \in\{1, \ldots, n\}$ such that for all

$$
i \in\{1, \ldots, n\} \backslash\{\sigma\}, c_{\sigma}>c_{i} .
$$

For each nonnegative integer $t$ let $x(t)=\left(x_{1}(t), \ldots, x_{n}(t)\right) \geq 0$ denote the amounts of the $n$ types of machines that are available in time-period $t$, and let $z(t+1)=\left(z_{1}(t+1), \ldots, z_{n}(t+\right.$ $1)) \geq 0$ be the gross investments in the $n$ types of machines during period $t+1$. Hence, $z(t+1)=(x(t+1)-x(t))+d x(t)$, the sum of net investment and of depreciation. Let $y(t)=$ $\left(y_{1}(t), \ldots, y_{n}(t)\right)$ be the amounts of the $n$ types of machines used for production of the consumption good, $b y(t)$, during period $t+1$. Let the total labor force of the economy be stationary and positive. We normalize it to be unity. It is clear that gross investment, $z(t+1)$ representing the production of new machines of the various types, requires $a z(t+1)$ units of labor in period $t$. Also $y(t)$ representing the use of available machines for manufacture of the consumption good, requires $e y(t)$ units of labor in period $t$. Thus, the availability of labor constrains employment in the consumption and investment sectors is descried by $a z(t+1)+e y(t) \leq 1$. Note that the flow of consumption and of investment (new machines) are in gestation during the period and available at the end of it. We now give a formal description of this technological structure.

A sequence $\{x(t), y(t)\}_{t=0}^{\infty}$ is called a program if, for each integer $t \geq 0$,

$$
\begin{gathered}
(x(t), y(t)) \in R_{+}^{n} \times R_{+}^{n}, x(t+1) \geq(1-d) x(t), 0 \leq y(t) \leq x(t), \\
a(x(t+1)-(1-d) x(t))+e y(t) \leq 1 .
\end{gathered}
$$

Let $T_{1}, T_{2}$ be integers such that $0 \leq T_{1}<T_{2}$. A pair of sequences

$$
\left(\{x(t)\}_{t=T_{1}}^{T_{2}},\{y(t)\}_{t=T_{1}}^{T_{2}-1}\right)
$$

is called a program if $x\left(T_{2}\right) \in R_{+}^{n}$ and, for each integer $t$ with $T_{1} \leq t<T_{2}$, relations (2.1) hold.

We associate with every program $\{x(t), y(t)\}_{t=0}^{\infty}$ its gross investment sequence $\{z(t+1)\}_{t=0}^{\infty}$ such that

$$
z(t+1)=x(t+1)-(1-d) x(t), t=0,1, \ldots
$$

and a consumption sequence $\{b y(t)\}_{t=0}^{\infty}$.

The results presented in this section were obtained in [19].

Proposition 2.1. For every program $\{x(t), y(t)\}_{t=0}^{\infty}$ there exists a constant $m(x(0))>0$, depending only on $x(0)$, such that $x(t) \leq m(x(0))$ for all nonnegative integers $t$.

Let $w:[0, \infty) \rightarrow R^{1}$ be a continuous strictly increasing concave and differentiable function which represents the preferences of the planner.

We use the following optimality criterion.

A program $\left\{x^{*}(t), y^{*}(t)\right\}_{t=0}^{\infty}$ is weakly optimal if

$$
\liminf _{T \rightarrow \infty} \sum_{t=0}^{T}\left[w(b y(t))-w\left(b y^{*}(t)\right)\right] \leq 0
$$

for every program $\{x(t), y(t)\}_{t=0}^{\infty}$ satisfying $x(0)=x^{*}(0)$.

Set

$$
\Omega=\left\{\left(x, x^{\prime}\right) \in R_{+}^{n} \times R_{+}^{n}: x^{\prime}-(1-d) x \geq 0\right.
$$


and $\left.a\left(x^{\prime}-(1-d) x\right) \leq 1\right\}$.

We have a correspondence $\Lambda: \Omega \rightarrow R_{+}^{n}$ given by

$$
\Lambda\left(x, x^{\prime}\right)=\left\{y \in R_{+}^{n}: 0 \leq y \leq x \text { and } e y \leq 1-a\left(x^{\prime}-(1-d) x\right)\right\},\left(x, x^{\prime}\right) \in \Omega .
$$

For any $\left(x, x^{\prime}\right) \in \Omega$, define

$$
u\left(x, x^{\prime}\right)=\max \left\{w(b y): y \in \Lambda\left(x, x^{\prime}\right)\right\} .
$$

A golden-rule stock is $\widehat{x} \in R_{+}^{n}$ such that $(\widehat{x}, \widehat{x})$ is a solution to the problem: maximize $u\left(x, x^{\prime}\right)$ subject to

(i) $x^{\prime} \geq x$; (ii) $\left(x, x^{\prime}\right) \in \Omega$.

For $i=1, \ldots, n$, set

$$
\widehat{q}_{i}=a_{i} b_{i} /\left(1+d a_{i}\right), \widehat{p}_{i}=w^{\prime}\left(b_{\sigma}\left(1+d a_{\sigma}\right)^{-1}\right) \widehat{q}_{i}, \widehat{y}=\left(1+d a_{\sigma}\right)^{-1} e(\sigma) .
$$

The following useful lemma plays an important role in our study.

Lemma 2.1. $w(b \widehat{y}) \geq w(b y)+\widehat{p} x^{\prime}-\widehat{p} x$ for any $\left(x, x^{\prime}\right) \in \Omega$ and for any $y \in \Lambda\left(x, x^{\prime}\right)$.

Theorem 2.1. There exists a unique golden-rule stock $\widehat{x}=\left(1+d a_{\sigma}\right)^{-1} e(\sigma)$.

We use the following notion of good programs introduced by Gale [13] and used in optimal control [9, 61, 76].

A program $\{x(t), y(t)\}_{t=0}^{\infty}$ is called good if there exists $M \in R^{1}$ such that

$$
\sum_{t=0}^{T}(w(b y(t))-w(b \widehat{y})) \geq M \text { for all integers } T \geq 0 .
$$

A program is called bad if

$$
\lim _{T \rightarrow \infty} \sum_{t=0}^{T}(w(b y(t))-w(b \widehat{y}))=-\infty .
$$

Proposition 2.2. Let $x_{0} \in R_{+}^{n}$. Then there exists a good program

$$
\{x(t), y(t)\}_{t=0}^{\infty}
$$

which satisfies $x(0)=x_{0}$.

Proposition 2.3. Let $\{x(t), y(t)\}_{t=0}^{\infty}$ be a program. Then there exists a constant $M(x(0)) \geq 0$ such that, for every pair of nonnegative integers $t_{1} \leq t_{2}$,

$$
\sum_{t=t_{1}}^{t_{2}}(w(b y(t))-w(b \widehat{y})) \leq M(x(0)) .
$$

Proposition 2.3 easily implies the following result.

Proposition 2.4. Every program, which is not good, is bad.

For any $\left(x, x^{\prime}\right) \in \Omega$ and any $y \in \Lambda\left(x, x^{\prime}\right)$, set

$$
\delta\left(x, y, x^{\prime}\right)=\widehat{p}\left(x-x^{\prime}\right)-(w(b y)-w(b \widehat{y})) .
$$

We say that a program $\{x(t), y(t)\}_{t=0}^{\infty}$ has the average turnpike property if

$$
\lim _{T \rightarrow \infty} T^{-1} \sum_{t=0}^{T-1}(x(t), y(t))=(\widehat{x}, \widehat{y}) .
$$


Proposition 2.5. Assume that a program $\{x(t), y(t)\}_{t=0}^{\infty}$ is good. Then it has the average turnpike property.

The next result easily follows from Lemma 2.1 and (2.2).

Proposition 2.6. Assume that $\{x(t), y(t)\}_{t=0}^{\infty}$ is a program. Then, for every integer $t \geq 0$,

$$
\delta(x(t), y(t), x(t+1)) \geq 0
$$

and, for every natural number $T$,

$$
\sum_{t=0}^{T}(w(b y(t))-w(b \widehat{y}))=\widehat{p}(x(0)-x(T+1))-\sum_{t=0}^{T} \delta(x(t), y(t), x(t+1)) .
$$

Proposition 2.6 implies the following result.

Proposition 2.7. A program $\{x(t), y(t)\}_{t=0}^{\infty}$ is good if and only if

$$
\sum_{t=0}^{\infty} \delta(x(t), y(t), x(t+1))<\infty .
$$

For every $x_{0} \in R_{+}^{n}$ define

$$
\Delta\left(x_{0}\right)=\inf \left\{\sum_{t=0}^{\infty} \delta(x(t), y(t), x(t+1)):\{x(t), y(t)\}_{t=0}^{\infty}\right.
$$

is a program such that $\left.x(0)=x_{0}\right\}$.

Proposition 2.8. Let $x_{0} \in R_{+}^{n}$. Then

$$
0 \leq \Delta\left(x_{0}\right)<\infty
$$

and there exists a program $\left\{x^{\prime}(t), y^{\prime}(t)\right\}_{t=0}^{\infty}$ such that

$$
x^{\prime}(0)=x_{0}, \Delta\left(x_{0}\right)=\sum_{t=0}^{\infty} \delta\left(x^{\prime}(t), y^{\prime}(t), x^{\prime}(t+1)\right) .
$$

Proposition 2.9. Assume that a program $\{x(t), y(t)\}_{t=0}^{\infty}$ satisfies

$$
\Delta(x(0))=\sum_{t=0}^{\infty} \delta(x(t), y(t), x(t+1))
$$

Then it is weakly optimal.

Propositions 2.8 and 2.9 and (2.2) imply the following result.

Theorem 2.2. Let $x_{0} \in R_{+}^{n}$. Then there exists a weakly optimal program $\{x(t), y(t)\}_{t=0}^{\infty}$ satisfying $x(0)=x_{0}$. If $x_{0}=\widehat{x}$, then the program $\{x(t), y(t)\}_{t=0}^{\infty}$ satisfying

$$
x(t)=y(t)=\widehat{x}, t=0,1, \ldots
$$

is weakly optimal.

The following auxiliary result plays an important role in our study of the RSS model. 
Lemma 2.2. Let

$$
\xi_{\sigma}=1-d-a_{\sigma}^{-1}
$$

The von Neumann facet

$$
\left\{\left(x, x^{\prime}\right) \in \Omega \text { : there exists } y \in \Lambda\left(x, x^{\prime}\right) \text { such that } \delta\left(x, y, x^{\prime}\right)=0\right\}
$$

is a subset of the set

$$
\left\{\left(x, x^{\prime}\right) \in \Omega: x_{i}^{\prime}=x_{i}=0, i \in\{1, \ldots, n\} \backslash\{\sigma\}, x_{\sigma}^{\prime}=a_{\sigma}^{-1}+\xi_{\sigma} x_{\sigma}\right\}
$$

with the equality if the function $w$ is linear. If the function $w$ is strictly concave, then the face is the singleton $\{(\widehat{x}, \widehat{x})\}$.

The next result easily follows from Proposition 2.2.

Proposition 2.10. Any weakly optimal program is good.

\section{OVERTAKING OPTIMAL PROGRAMS}

In this section we continue to use the assumptions introduced in Section 2. The following three theorems were obtained in [59].

Theorem 3.1. Assume that the function $w$ is strictly concave. Then, for every good program $\{x(t), y(t)\}_{t=0}^{\infty}$,

$$
\lim _{t \rightarrow \infty}(x(t), y(t))=(\widehat{x}, \widehat{x}) .
$$

Set

$$
\xi_{\sigma}=1-d-\left(1 / a_{\sigma}\right) .
$$

Theorem 3.2. Assume that $\xi_{\sigma} \neq-1$. Then

$$
\lim _{t \rightarrow \infty}(x(t), y(t))=(\widehat{x}, \widehat{x})
$$

for every good program $\{x(t), y(t)\}_{t=0}^{\infty}$.

In this paper we use a notion of an overtaking optimal program introduced by Gale [13], von Weizsacker [58]. This optimality criterion is used in optimal control [9, 61, 76].

A program $\left\{x^{*}(t), y^{*}(t)\right\}_{t=0}^{\infty}$ is overtaking optimal if

$$
\limsup _{T \rightarrow \infty} \sum_{t=0}^{T}\left[w(b y(t))-w\left(b y^{*}(t)\right)\right] \leq 0
$$

for every program $\{x(t), y(t)\}_{t=0}^{\infty}$, which satisfies $x(0)=x^{*}(0)$.

Theorem 3.3. Assume that for every good program $\{x(t), y(t)\}_{t=0}^{\infty}$,

$$
\lim _{t \rightarrow \infty}(x(t), y(t))=(\widehat{x}, \widehat{x}) .
$$

Then, for every point $x_{0} \in R_{+}^{n}$, there is an overtaking optimal program $\{x(t), y(t)\}_{t=0}^{\infty}$ such that $x(0)=x_{0}$.

Corollary 3.1. Assume that the function $w$ is strictly concave. Then, for every point $x_{0} \in R_{+}^{n}$, there exists an overtaking optimal program $\{x(t), y(t)\}_{t=0}^{\infty}$ satisfying $x(0)=x_{0}$.

Corollary 3.2. Assume that $\xi_{\sigma} \neq-1$. Then, for every point $x_{0} \in R_{+}^{n}$, there is an overtaking optimal program $\{x(t), y(t)\}_{t=0}^{\infty}$ such that $x(0)=x_{0}$. 
The following three theorems were obtained in [30].

Theorem 3.4. Assume that for each good program $\{u(t), v(t)\}_{t=0}^{\infty}$,

$$
\lim _{t \rightarrow \infty}(u(t), v(t))=(\widehat{x}, \widehat{x}) .
$$

Then, for each program $\{x(t), y(t)\}_{t=0}^{\infty}$, the following conditions are equivalent:

(i) $\sum_{t=0}^{\infty} \delta(x(t), y(t), x(t+1))=\Delta(x(0))$.

(ii) $\{x(t), y(t)\}_{t=0}^{\infty}$ is overtaking optimal.

(iii) $\{x(t), y(t)\}_{t=0}^{\infty}$ is weakly optimal.

Theorem 3.5. Assume that at least one of the following conditions holds:

(a) $w$ is strictly concave.

(b) $\xi_{\sigma} \neq-1$.

Let $M_{0}, \varepsilon>0$. Then there exists a natural number $T_{0}$ such that for each overtaking optimal program $\{x(t), y(t)\}_{t=0}^{\infty}$ satisfying $x(0) \leq M_{0}$ e and each integer $t \geq T_{0}$,

$$
\|x(t)-\widehat{x}\|,\|y(t)-\widehat{x}\| \leq \varepsilon .
$$

Theorem 3.6. Assume that at least one of the following conditions holds:

(a) $w$ is strictly concave.

(b) $\xi_{\sigma} \neq-1$.

Let $\varepsilon>0$. Then there is $\delta>0$ such that for each overtaking optimal program $\{x(t), y(t)\}_{t=0}^{\infty}$ satisfying $\|x(0)-\widehat{x}\| \leq \delta$ the following inequality holds:

$$
\|x(t)-\widehat{x}\|,\|y(t)-\widehat{x}\| \leq \varepsilon
$$

for all integers $t \geq 0$.

In [62], we studied the structure of good programs of the RSS model and proved the following three results.

Theorem 3.7. Let a program $\{x(t), y(t)\}_{t=0}^{\infty}$ be good. Then, for each $i \in\{1, \ldots, n\} \backslash\{\sigma\}$,

$$
\begin{gathered}
\sum_{t=0}^{\infty} x_{i}(t)<\infty, \\
\sum_{t=0}^{\infty}\left(x_{\sigma}(t)-y_{\sigma}(t)\right)<\infty
\end{gathered}
$$

and the sequence $\left\{\sum_{t=0}^{T-1} x_{\sigma}(t)-T\left(1+d a_{\sigma}\right)^{-1}\right\}_{T=1}^{\infty}$ is bounded.

Theorem 3.8. Let the function $w$ be linear. Then a program $\{x(t), y(t)\}_{t=0}^{\infty}$ is good if and only if, for each $i \in\{1, \ldots, n\} \backslash\{\sigma\}$,

$$
\begin{gathered}
\sum_{t=0}^{\infty} x_{i}(t)<\infty, \\
\sum_{t=0}^{\infty}\left(x_{\sigma}(t)-y_{\sigma}(t)\right)<\infty
\end{gathered}
$$

and the sequence $\left\{\sum_{t=0}^{T-1} x_{\sigma}(t)-T\left(1+d a_{\sigma}\right)^{-1}\right\}_{T=1}^{\infty}$ is bounded. 
Theorem 3.9. Let $w \in C^{2}, w^{\prime \prime}(b \widehat{x}) \neq 0$ and let for every good program $\{u(t), v(t)\}_{t=0}^{\infty}$,

$$
\lim _{t \rightarrow \infty}(u(t), v(t))=(\widehat{x}, \widehat{x}) .
$$

Then a program $\{x(t), y(t)\}_{t=0}^{\infty}$ is good if and only if, for each $i \in\{1, \ldots, n\} \backslash\{\sigma\}$,

$$
\begin{gathered}
\sum_{t=0}^{\infty} x_{i}(t)<\infty, \\
\sum_{t=0}^{\infty}\left(x_{\sigma}(t)-y_{\sigma}(t)\right)<\infty \\
\sum_{t=0}^{\infty}\left(y_{\sigma}(t)-\widehat{x}_{\sigma}\right)^{2}<\infty
\end{gathered}
$$

and the sequence $\left\{\sum_{t=0}^{T-1} x_{\sigma}(t)-T\left(1+d a_{\sigma}\right)^{-1}\right\}_{T=1}^{\infty}$ is bounded.

\section{TURNPIKE PROPERTIES}

In this section we discuss the turnpike properties for the Robinson-Solow-Srinivasan model. To have these properties means that the approximate solutions of the problems are essentially independent of the choice of an interval and endpoint conditions. We show that these turnpike properties hold and that they are stable under perturbations of an objective function.

We continue to use the assumptions introduced in Section 2.

Let $z \in R_{+}^{n}$ and $T \geq 1$ be a natural number. Set

$$
U(z, T)=\sup \left\{\sum_{t=0}^{T-1} w(b y(t)):\left(\{x(t)\}_{t=0}^{T},\{y(t)\}_{t=0}^{T-1}\right)\right.
$$

is a program such that $x(0)=z\}$.

Clearly, $U(z, T)$ is a finite number. Let $x_{0}, x_{1} \in R_{+}^{n}, T_{1}, T_{2}$ be integers, and $0 \leq T_{1}<T_{2}$. Define

$$
U\left(x_{0}, x_{1}, T_{1}, T_{2}\right)=\sup \left\{\sum_{t=T_{1}}^{T_{2}-1} w(b y(t)):\left(\{x(t)\}_{t=T_{1}}^{T_{2}},\{y(t)\}_{t=T_{1}}^{T_{2}-1}\right)\right.
$$

is a program such that $\left.x\left(T_{1}\right)=x_{0}, x\left(T_{2}\right) \geq x_{1}\right\}$.

(Here we suppose that a supremum over empty set is $-\infty$.) Clearly,

$$
U\left(x_{0}, x_{1}, T_{1}, T_{2}\right)<\infty .
$$

It is also clear that, for any $z \in R_{+}^{n}$ and any integer $T \geq 1, U(z, T)=U(z, 0,0, T)$.

In this section we assume that the following asymptotic turnpike property holds:

(ATP) Each good program $\{x(t), y(t)\}_{t=0}^{\infty}$ converges to the golden-rule stock $(\widehat{x}, \widehat{x})$ :

$$
\lim _{t \rightarrow \infty}(x(t), y(t))=(\widehat{x}, \widehat{x}) .
$$

With $\operatorname{Card}(A)$, we denote in the sequel the cardinality of a finite set $A$.

The following two turnpike results were obtained in [32]. 
Theorem 4.1. Let $M, \varepsilon$ be positive numbers and $\Gamma \in(0,1)$. Then there exists a natural number $L$ such that for each integer $T>L$, each $z_{0}, z_{1} \in R_{+}^{n}$ satisfying $z_{0} \leq M e$ and $a z_{1} \leq \Gamma d^{-1}$ and each program $\left(\{x(t)\}_{t=0}^{T},\{y(t)\}_{t=0}^{T-1}\right)$ which satisfies

$$
x(0)=z_{0}, x(T) \geq z_{1}, \quad \sum_{t=0}^{T-1} w(b y(t)) \geq U\left(z_{0}, z_{1}, 0, T\right)-M,
$$

the following inequality holds:

$$
\operatorname{Card}\{i \in\{0, \ldots, T-1\}: \max \{\|x(t)-\widehat{x}\|,\|y(t)-\widehat{x}\|\}>\varepsilon\} \leq L .
$$

Theorem 4.2. Let $M, \varepsilon$ be positive numbers and $\Gamma \in(0,1)$. Then there exist a natural number $L$ and a positive number $\gamma$ such that for each integer $T>2 L$, each $z_{0}, z_{1} \in R_{+}^{n}$ satisfying $z_{0} \leq M e$ and $a z_{1} \leq \Gamma d^{-1}$ and each program $\left(\{x(t)\}_{t=0}^{T},\{y(t)\}_{t=0}^{T-1}\right)$ which satisfies

$$
x(0)=z_{0}, x(T) \geq z_{1}, \quad \sum_{t=0}^{T-1} w(b y(t)) \geq U\left(z_{0}, z_{1}, 0, T\right)-\gamma,
$$

there are integers $\tau_{1}, \tau_{2}$ such that

$$
\begin{gathered}
\tau_{1} \in[0, L], \tau_{2} \in[T-L, T], \\
\|x(t)-\widehat{x}\|,\|y(t)-\widehat{x}\| \leq \varepsilon \text { for all } t=\tau_{1}, \ldots, \tau_{2}-1 .
\end{gathered}
$$

Moreover if $\|x(0)-\widehat{x}\| \leq \gamma$, then $\tau_{1}=0$ and if $\|x(T)-\widehat{x}\| \leq \gamma$, then $\tau_{2}=T$.

In [70], we continued to study the turnpike phenomenon for the RSS model and proved the following three turnpike results which are extensions of Theorem 4.2.

Theorem 4.3. Suppose that for each good program $\{u(t), v(t)\}_{t=0}^{\infty}$,

$$
\lim _{t \rightarrow \infty}(u(t), v(t))=(\widehat{x}, \widehat{x}) .
$$

Let $M, \varepsilon$ be positive numbers and $\Gamma \in(0,1)$. Then there exist a natural number $L$ and a positive number $\gamma$ such that for each integer $T>2 L$, each $z_{0}, z_{1} \in R_{+}^{n}$ satisfying $z_{0} \leq M e$ and $a z_{1} \leq \Gamma d^{-1}$ and each program $\left(\{x(t)\}_{t=0}^{T},\{y(t)\}_{t=0}^{T-1}\right)$ which satisfies

$$
x(0)=z_{0}, x(T) \geq z_{1},
$$

$$
\sum_{t=\tau}^{\tau+L-1} w(b y(t)) \geq U(x(\tau), x(\tau+L), 0, L)-\gamma \text { for all } \tau \in\{0, \ldots, T-L\}
$$

and

$$
\sum_{t=T-L}^{T-1} w(b y(t)) \geq U\left(x(T-L), z_{1}, 0, L\right)-\gamma
$$

there are integers $\tau_{1}, \tau_{2}$ such that

$$
\begin{gathered}
\tau_{1} \in[0, L], \tau_{2} \in[T-L, T], \\
\|x(t)-\widehat{x}\|,\|y(t)-\widehat{x}\| \leq \varepsilon \text { for all } t=\tau_{1}, \ldots, \tau_{2}-1 .
\end{gathered}
$$

Moreover if $\|x(0)-\widehat{x}\| \leq \gamma$, then $\tau_{1}=0$ and if $\|x(T)-\widehat{x}\| \leq \gamma$, then $\tau_{2}=T$. 
Theorem 4.4. Suppose that for each good program $\{u(t), v(t)\}_{t=0}^{\infty}$,

$$
\lim _{t \rightarrow \infty}(u(t), v(t))=(\widehat{x}, \widehat{x}) .
$$

Let $M, \varepsilon$ be positive numbers. Then there exist a natural number $L$ and a positive number $\gamma$ such that for each integer $T>2 L$, each $z_{0} \in R_{+}^{n}$ satisfying $z_{0} \leq$ Me and each program $\left(\{x(t)\}_{t=0}^{T},\{y(t)\}_{t=0}^{T-1}\right)$ which satisfies

$$
x(0)=z_{0},
$$

$$
\sum_{t=\tau}^{\tau+L-1} w(b y(t)) \geq U(x(\tau), x(\tau+L), 0, L)-\gamma \text { for all } \tau \in\{0, \ldots, T-L\}
$$

and

$$
\sum_{t=T-L}^{T-1} w(b y(t)) \geq U(x(T-L), L)-\gamma,
$$

there are integers $\tau_{1}, \tau_{2}$ such that

$$
\begin{gathered}
\tau_{1} \in[0, L], \tau_{2} \in[T-L, T], \\
\|x(t)-\widehat{x}\|,\|y(t)-\widehat{x}\| \leq \varepsilon \text { for all } t=\tau_{1}, \ldots, \tau_{2}-1 .
\end{gathered}
$$

Moreover if $\|x(0)-\widehat{x}\| \leq \gamma$ then $\tau_{1}=0$ and if $\|x(T)-\widehat{x}\| \leq \gamma$ then $\tau_{2}=T$.

Theorem 4.5. Suppose that for each good program $\{u(t), v(t)\}_{t=0}^{\infty}$,

$$
\lim _{t \rightarrow \infty}(u(t), v(t))=(\widehat{x}, \widehat{x}) .
$$

Let $M, \varepsilon$ be positive numbers. Then there exist a natural number $L$ and a positive number $\gamma$ such that for each integer $T>2 L$, each $z_{0} \in R_{+}^{n}$ satisfying $z_{0} \leq$ Me and each program $\left(\{x(t)\}_{t=0}^{T},\{y(t)\}_{t=0}^{T-1}\right)$ which satisfies

$$
\begin{gathered}
x(0)=z_{0}, \\
\sum_{t=0}^{T-1} w(b y(t)) \geq U\left(z_{0}, T\right)-\gamma
\end{gathered}
$$

there are integers $\tau_{1}, \tau_{2}$ such that

$$
\begin{gathered}
\tau_{1} \in[0, L], \tau_{2} \in[T-L, T] \\
\|x(t)-\widehat{x}\|,\|y(t)-\widehat{x}\| \leq \varepsilon \text { for all } t=\tau_{1}, \ldots, \tau_{2}-1 .
\end{gathered}
$$

Moreover if $\|x(0)-\widehat{x}\| \leq \gamma$, then $\tau_{1}=0$ and $\|x(T)-\widehat{x}\| \leq \gamma$, then $\tau_{2}=T$.

For every positive number $M$ and every function $\phi: R_{+}^{n} \rightarrow R^{1}$, define

$$
\|\phi\|_{M}=\sup \left\{|\phi(z)|: z \in R^{n} \text { and } 0 \leq z \leq M e\right\} .
$$

Let integers $T_{1}, T_{2}$ satisfy $0 \leq T_{1}<T_{2}$, and $w_{i}: R_{+}^{n} \rightarrow R^{1}, i=T_{1}, \ldots, T_{2}-1$ be bounded on bounded subsets of $R_{+}^{n}$ functions. For every pair of points $z_{0}, z_{1} \in R_{+}^{n}$, define

$$
U\left(\left\{w_{t}\right\}_{t=T_{1}}^{T_{2}-1}, z_{0}, z_{1}\right)=\sup \left\{\sum_{t=T_{1}}^{T_{2}-1} w_{t}(y(t)):\right.
$$

$$
\left.\left(\{x(t)\}_{t=T_{1}}^{T_{2}},\{y(t)\}_{t=T_{1}}^{T_{2}-1}\right) \text { is a program such that } x\left(T_{1}\right)=z_{0}, x\left(T_{2}\right) \geq z_{1}\right\},
$$




$$
\begin{gathered}
U\left(\left\{w_{t}\right\}_{t=T_{1}}^{T_{2}-1}, z_{0}\right)=\sup \left\{\sum_{t=T_{1}}^{T_{2}-1} w_{t}(y(t)):\right. \\
\left.\left(\{x(t)\}_{t=T_{1}}^{T_{2}},\{y(t)\}_{t=T_{1}}^{T_{2}-1}\right) \text { is a program such that } x\left(T_{1}\right)=z_{0}\right\} .
\end{gathered}
$$

(Here we assume that supremum over empty set is $-\infty$.) It is not difficult to see that the following result holds.

Lemma 4.1. Let integers $T_{1}, T_{2}$ satisfy $0 \leq T_{1}<T_{2}$ and $w_{i}: R_{+}^{n} \rightarrow R^{1}, i=T_{1}, \ldots, T_{2}-1$ be bounded on bounded subsets of $R_{+}^{n}$ upper semicontinuous functions. Then the following assertions hold.

1. For every point $z_{0} \in R_{+}^{n}$, there exists a program $\left(\{x(t)\}_{t=T_{1}}^{T_{2}},\{y(t)\}_{T_{1}}^{T_{2}-1}\right)$ such that

$$
x\left(T_{1}\right)=z_{0}, \sum_{t=T_{1}}^{T_{2}-1} w_{t}(y(t))=U\left(\left\{w_{t}\right\}_{t=T_{1}}^{T_{2}-1}, z_{0}\right) .
$$

2. For every pair of points $z_{0}, z_{1} \in R_{+}^{n}$ such that $U\left(\left\{w_{t}\right\}_{t=T_{1}}^{T_{2}-1}, z_{0}, z_{1}\right)$ is finite, there exists a program $\left(\{x(t)\}_{t=T_{1}}^{T_{2}},\{y(t)\}_{t=T_{1}}^{T_{2}-1}\right)$ such that $x(0)=z_{0}, x\left(T_{2}\right) \geq z_{1}$ and

$$
\sum_{t=T_{1}}^{T_{2}-1} w_{t}(y(t))=U\left(\left\{w_{t}\right\}_{t=T_{1}}^{T_{2}-1}, z_{0}, z_{1}\right) .
$$

The following stability results were obtained in [70]. It was proved that the turnpike phenomenon is stable under small perturbations of the utility functions.

Theorem 4.6. Suppose that for each good program $\{u(t), v(t)\}_{t=0}^{\infty}$,

$$
\lim _{t \rightarrow \infty}(u(t), v(t))=(\widehat{x}, \widehat{x}) .
$$

Let $M>\max \left\{\left(a_{i} d\right)^{-1}: i=1, \ldots, n\right\}, \varepsilon>0$ and $\Gamma \in(0,1)$. Then there exist a natural number $L$ and a positive number $\tilde{\gamma}$ such that for each integer $T>2 L$, each $z_{0}, z_{1} \in R_{+}^{n}$ satisfying $z_{0} \leq M e$ and $a z_{1} \leq \Gamma d^{-1}$, each finite sequence of functions $w_{i}: R_{+}^{n} \rightarrow R^{1}, i=0, \ldots, T-1$ which are bounded on bounded subsets of $R_{+}^{n}$ and such that

$$
\left\|w_{i}-w(b(\cdot))\right\|_{M} \leq \tilde{\gamma}
$$

for every integer $i \in\{0, \ldots, T-1\}$ and every program $\left(\{x(t)\}_{t=0}^{T},\{y(t)\}_{t=0}^{T-1}\right)$ such that

$$
\begin{gathered}
x(0)=z_{0}, x(T) \geq z_{1}, \\
\sum_{t=\tau}^{\tau+L-1} w_{t}(y(t)) \geq U\left(\left\{w_{t}\right\}_{t=\tau}^{\tau+L-1}, x(\tau), x(\tau+L)\right)-\tilde{\gamma}
\end{gathered}
$$

for every $\tau \in\{0, \ldots, T-L\}$ and

$$
\sum_{t=T-L}^{T-1} w_{t}(y(t)) \geq U\left(\left\{w_{t}\right\}_{t=T-L}^{T-1}, x(T-L), z_{1}\right)-\tilde{\gamma},
$$

there exist integers $\tau_{1}, \tau_{2}$ such that

$$
\begin{gathered}
\tau_{1} \in[0, L], \tau_{2} \in[T-L, T], \\
\|x(t)-\widehat{x}\|,\|y(t)-\widehat{x}\| \leq \varepsilon \text { for all } t=\tau_{1}, \ldots, \tau_{2}-1 .
\end{gathered}
$$

Moreover if $\mid x(0)-\widehat{x} \| \leq \tilde{\gamma}$, then $\tau_{1}=0$ and if $\|x(T)-\widehat{x}\| \leq \tilde{\gamma}$, then $\tau_{2}=T$. 
Theorem 4.7. Suppose that for each good program $\{u(t), v(t)\}_{t=0}^{\infty}$,

$$
\lim _{t \rightarrow \infty}(u(t), v(t))=(\widehat{x}, \widehat{x}) .
$$

Let $M>\max \left\{\left(a_{i} d\right)^{-1}: i=1, \ldots, n\right\}$ and $\varepsilon>0$. Then there exist a natural number $L$ and $a$ positive number $\tilde{\gamma}$ such that for each integer $T>2 L$, each $z_{0} \in R_{+}^{n}$ satisfying $z_{0} \leq$ Me, each finite sequence of functions $w_{i}: R_{+}^{n} \rightarrow R^{1}, i=0, \ldots, T-1$ which are bounded on bounded subsets of $R_{+}^{n}$ and such that

$$
\left\|w_{i}-w(b(\cdot))\right\|_{M} \leq \tilde{\gamma}
$$

for each $i \in\{0, \ldots, T-1\}$ and each program $\left(\{x(t)\}_{t=0}^{T},\{y(t)\}_{t=0}^{T-1}\right)$ which satisfies

$$
\begin{gathered}
x(0)=z_{0}, \\
\sum_{t=\tau}^{\tau+L-1} w_{t}(y(t)) \geq U\left(\left\{w_{t}\right\}_{t=\tau}^{\tau+L-1}, x(\tau), x(\tau+L)\right)-\tilde{\gamma}
\end{gathered}
$$

for each integer $\tau \in\{0, \ldots, T-L\}$ and

$$
\sum_{t=T-L}^{T-1} w_{t}(y(t)) \geq U\left(\left\{w_{t}\right\}_{t=T-L}^{T-1}, x(T-L)\right)-\tilde{\gamma},
$$

there are integers $\tau_{1}, \tau_{2}$ such that

$$
\begin{gathered}
\tau_{1} \in[0, L], \tau_{2} \in[T-L, T] \\
\|x(t)-\widehat{x}\|,\|y(t)-\widehat{x}\| \leq \varepsilon \text { for all } t=\tau_{1}, \ldots, \tau_{2}-1 .
\end{gathered}
$$

Moreover if $\|x(0)-\widehat{x}\| \leq \gamma$, then $\tau_{1}=0$ and if $\|x(T)-\widehat{x}\| \leq \gamma$, then $\tau_{2}=T$.

Theorem 4.8. Suppose that for each good program $\{u(t), v(t)\}_{t=0}^{\infty}$,

$$
\lim _{t \rightarrow \infty}(u(t), v(t))=(\widehat{x}, \widehat{x}) .
$$

Let $M>\max \left\{\left(a_{i} d\right)^{-1}: i=1, \ldots, n\right\}, \varepsilon>0$ and $\Gamma \in(0,1)$. Then there exist a natural number $L$, a positive number $\gamma$ and $\lambda>1$ such that for each integer $T>2 L$, each $z_{0}, z_{1} \in R_{+}^{n}$ satisfying $z_{0} \leq$ Me and $a z_{1} \leq \Gamma d^{-1}$, each finite sequence of functions $w_{i}: R_{+}^{n} \rightarrow R^{1}, i=0, \ldots, T-1$ which are bounded on bounded subsets of $R_{+}^{n}$ and such that $\left\|w_{i}-w(b(\cdot))\right\|_{M} \leq \gamma$ for each $i \in$ $\{0, \ldots, T-1\}$, each sequence $\left\{\alpha_{i}\right\}_{i=0}^{T-1} \subset(0,1]$ such that for each $i, j \in\{0, \ldots, T-1\}$ satisfying $|j-i| \leq L$ the inequality $\alpha_{i} \alpha_{j}^{-1} \leq \lambda$ holds and each program $\left(\{x(t)\}_{t=0}^{T},\{y(t)\}_{t=0}^{T-1}\right)$ such that

$$
\begin{gathered}
x(0)=z_{0}, x(T) \geq z_{1}, \\
\sum_{t=\tau}^{\tau+L-1} \alpha_{t} w_{t}(y(t)) \geq U\left(\left\{\alpha_{t} w_{t}\right\}_{t=\tau}^{\tau+L-1}, x(\tau), x(\tau+L)\right)-\gamma \alpha_{\tau}
\end{gathered}
$$

for each integer $\tau \in\{0, \ldots, T-L\}$ and

$$
\sum_{t=T-L}^{T-1} \alpha_{t} w_{t}(y(t)) \geq U\left(\left\{\alpha_{t} w_{t}\right\}_{t=T-L}^{T-1}, z_{1}\right)-\gamma \alpha_{T-L}
$$

there are integers $\tau_{1}, \tau_{2}$ such that

$$
\begin{gathered}
\tau_{1} \in[0, L], \tau_{2} \in[T-L, T] \\
\|x(t)-\widehat{x}\|,\|y(t)-\widehat{x}\| \leq \varepsilon \text { for all } t=\tau_{1}, \ldots, \tau_{2}-1 .
\end{gathered}
$$

Moreover if $\|x(0)-\widehat{x}\| \leq \gamma$, then $\tau_{1}=0$ and if $\|x(0)-\widehat{x}\| \leq \gamma$, then $\tau_{2}=T$. 
Theorem 4.9. Suppose that for each good program $\{u(t), v(t)\}_{t=0}^{\infty}$,

$$
\lim _{t \rightarrow \infty}(u(t), v(t))=(\widehat{x}, \widehat{x}) .
$$

Let $M>\max \left\{\left(a_{i} d\right)^{-1}: i=1, \ldots, n\right\}$ and $\varepsilon>0$. Then there exist a natural number $L$, a positive number $\gamma$ and $\lambda>1$ such that for each integer $T>2 L$, each $z_{0} \in R_{+}^{n}$ satisfying $z_{0} \leq M e$, each finite sequence of functions $w_{i}: R_{+}^{n} \rightarrow R^{1}, i=0, \ldots, T-1$ which are bounded on bounded subsets of $R_{+}^{n}$ and such that

$$
\left\|w_{i}-w(b(\cdot))\right\|_{M} \leq \gamma
$$

for each $i \in\{0, \ldots, T-1\}$, each sequence $\left\{\alpha_{i}\right\}_{i=0}^{T-1} \subset(0,1]$ such that for each

$$
i, j \in\{0, \ldots, T-1\}
$$

satisfying $|j-i| \leq L$ the inequality $\alpha_{i} \alpha_{j}^{-1} \leq \lambda$ holds and each program $\left(\{x(t)\}_{t=0}^{T},\{y(t)\}_{t=0}^{T-1}\right)$ such that

$$
\begin{gathered}
x(0)=z_{0}, \\
\sum_{t=\tau}^{\tau+L-1} \alpha_{t} w_{t}(y(t)) \geq U\left(\left\{\alpha_{t} w_{t}\right\}_{t=\tau}^{\tau+L-1}, x(\tau), x(\tau+L)\right)-\gamma \alpha_{\tau}
\end{gathered}
$$

for each integer $\tau \in\{0, \ldots, T-L\}$ and

$$
\sum_{t=T-L}^{T-1} \alpha_{t} w_{t}(y(t)) \geq U\left(\left\{\alpha_{t} w_{t}\right\}_{t=T-L}^{T-1}, x(T-L)\right)-\gamma \alpha_{T-L}
$$

there are integers $\tau_{1}, \tau_{2}$ such that

$$
\begin{gathered}
\tau_{1} \in[0, L], \tau_{2} \in[T-L, T] \\
\|x(t)-\widehat{x}\|,\|y(t)-\widehat{x}\| \leq \varepsilon \text { for all } t=\tau_{1}, \ldots, \tau_{2}-1 .
\end{gathered}
$$

Moreover if $\|x(0)-\widehat{x}\| \leq \gamma$, then $\tau_{1}=0$ and if $\|x(T)-\widehat{x}\| \leq \gamma$, then $\tau_{2}=T$.

Theorem 4.10. Suppose that for each good program $\{u(t), v(t)\}_{t=0}^{\infty}$,

$$
\lim _{t \rightarrow \infty}(u(t), v(t))=(\widehat{x}, \widehat{x}) \text {. }
$$

Let $M>\max \left\{\left(a_{i} d\right)^{-1}: i=1, \ldots, n\right\}$ and $\varepsilon>0$. Then there exist a natural number $L$, a positive number $\gamma$ and $\lambda>1$ such that for each integer $T>2 L$, each $z_{0} \in R_{+}^{n}$ satisfying $z_{0} \leq M e$, each finite sequence of upper semicontinuous functions $w_{i}: R_{+}^{n} \rightarrow R^{1}, i=0, \ldots, T-1$ which are bounded on bounded subsets of $R_{+}^{n}$ and such that

$$
\left\|w_{i}-w(b(\cdot))\right\|_{M} \leq \gamma
$$

for each $i \in\{0, \ldots, T-1\}$, each sequence $\left\{\alpha_{i}\right\}_{i=0}^{T-1} \subset(0,1]$ such that for each

$$
i, j \in\{0, \ldots, T-1\}
$$

satisfying $|j-i| \leq L$ the inequality $\alpha_{i} \alpha_{j}^{-1} \leq \lambda$ holds and each program $\left(\{x(t)\}_{t=0}^{T},\{y(t)\}_{t=0}^{T-1}\right)$ such that

$$
x(0)=z_{0}
$$

and

$$
\sum_{t=0}^{T-1} \alpha_{t} w_{t}(y(t))=U\left(\left\{\alpha_{t} w_{t}\right\}_{t=0}^{T-1}, x(0)\right)
$$


there are integers $\tau_{1}, \tau_{2}$ such that

$$
\begin{gathered}
\tau_{1} \in[0, L], \tau_{2} \in[T-L, T] \\
\|x(t)-\widehat{x}\|,\|y(t)-\widehat{x}\| \leq \varepsilon \text { for all } t=\tau_{1}, \ldots, \tau_{2}-1 .
\end{gathered}
$$

Moreover if $\|x(0)-\widehat{x}\| \leq \gamma$, then $\tau_{1}=0$ and if $\|x(T)-\widehat{x}\| \leq \gamma$, then $\tau_{2}=T$.

Theorem 4.11. Suppose that for each good program $\{u(t), v(t)\}_{t=0}^{\infty}$,

$$
\lim _{t \rightarrow \infty}(u(t), v(t))=(\widehat{x}, \widehat{x}) .
$$

Let $M>\max \left\{\left(a_{i} d\right)^{-1}: i=1, \ldots, n\right\}, \varepsilon>0$ and $\Gamma \in(0,1)$. Then there exist a natural number $L$ and a positive number $\tilde{\gamma}$ such that for each integer $T>2 L$, each $z_{0}, z_{1} \in R_{+}^{n}$ satisfying $z_{0} \leq M e$ and $a z_{1} \leq \Gamma d^{-1}$, each finite sequence of functions $w_{i}: R_{+}^{n} \rightarrow R^{1}, i=0, \ldots, T-1$ which are bounded on bounded subsets of $R_{+}^{n}$ and such that

$$
\left\|w_{i}-w(b(\cdot))\right\|_{M} \leq \tilde{\gamma}
$$

for each $i \in\{0, \ldots, T-1\}$ and each program $\left(\{x(t)\}_{t=0}^{T},\{y(t)\}_{t=0}^{T-1}\right)$ such that

$$
\begin{gathered}
x(0)=z_{0}, x(T) \geq z_{1}, \\
\sum_{t=0}^{T-1} w_{t}(y(t)) \geq U\left(\left\{w_{t}\right\}_{t=0}^{T-1}, z_{0}, z_{1}\right)-\tilde{\gamma},
\end{gathered}
$$

there are integers $\tau_{1}, \tau_{2}$ such that

$$
\begin{gathered}
\tau_{1} \in[0, L], \tau_{2} \in[T-L, T], \\
\|x(t)-\widehat{x}\|,\|y(t)-\widehat{x}\| \leq \varepsilon \text { for all } t=\tau_{1}, \ldots, \tau_{2}-1 .
\end{gathered}
$$

Moreover if $\|x(0)-\widehat{x}\| \leq \tilde{\gamma}$, then $\tau_{1}=0$ and if $\|x(T)-\widehat{x}\| \leq \tilde{\gamma}$, then $\tau_{2}=T$.

Theorem 4.12. Suppose that for each good program $\{u(t), v(t)\}_{t=0}^{\infty}$,

$$
\lim _{t \rightarrow \infty}(u(t), v(t))=(\widehat{x}, \widehat{x}) .
$$

Let $M>\max \left\{\left(a_{i} d\right)^{-1}: i=1, \ldots, n\right\}$ and $\varepsilon>0$. Then there exist a natural number $L$ and $a$ positive number $\tilde{\gamma}$ such that for each integer $T>2 L$, each $z_{0} \in R_{+}^{n}$ satisfying $z_{0} \leq M e$, each finite sequence of functions $w_{i}: R_{+}^{n} \rightarrow R^{1}, i=0, \ldots, T-1$ which are bounded on bounded subsets of $R_{+}^{n}$ and such that

$$
\left\|w_{i}-w(b(\cdot))\right\|_{M} \leq \tilde{\gamma}
$$

for each $i \in\{0, \ldots, T-1\}$ and each program $\left(\{x(t)\}_{t=0}^{T},\{y(t)\}_{t=0}^{T-1}\right)$ which satisfies

$$
\begin{gathered}
x(0)=z_{0}, \\
\sum_{t=0}^{T-1} w_{t}(y(t)) \geq U\left(\left\{w_{t}\right\}_{t=0}^{T-1}, z_{0}\right)-\tilde{\gamma},
\end{gathered}
$$

there are integers $\tau_{1}, \tau_{2}$ such that

$$
\begin{gathered}
\tau_{1} \in[0, L], \tau_{2} \in[T-L, T], \\
\|x(t)-\widehat{x}\|,\|y(t)-\widehat{x}\| \leq \varepsilon \text { for all } t=\tau_{1}, \ldots, \tau_{2}-1 .
\end{gathered}
$$

Moreover if $\|x(0)-\widehat{x}\| \leq \tilde{\gamma}$, then $\tau_{1}=0$ and if $\|x(T)-\widehat{x}\| \leq \tilde{\gamma}$, then $\tau_{2}=T$.

The following results were obtained in [73]. 
Theorem 4.13. Suppose that for each good program $\{u(t), v(t)\}_{t=0}^{\infty}$,

$$
\lim _{t \rightarrow \infty}(u(t), v(t))=(\widehat{x}, \widehat{x}) .
$$

Let $M>\max \left\{\left(a_{i} d\right)^{-1}: i=1, \ldots, n\right\}, M_{0}>0, \varepsilon>0$ and $\Gamma \in(0,1)$. Then there exist a natural number $L$ and a positive number $\tilde{\gamma}$ such that for each integer $T>L$, each $z_{0}, z_{1} \in R_{+}^{n}$ satisfying $z_{0} \leq$ Me and $a z_{1} \leq \Gamma d^{-1}$, each finite sequence of functions $w_{i}: R_{+}^{n} \rightarrow R^{1}, i=0, \ldots, T-1$ which are bounded on bounded subsets of $R_{+}^{n}$ and such that

$$
\left\|w_{i}-w(b(\cdot))\right\|_{M} \leq \tilde{\gamma}
$$

for each $i \in\{0, \ldots, T-1\}$ and each program $\left(\{x(t)\}_{t=0}^{T},\{y(t)\}_{t=0}^{T-1}\right)$ such that

$$
\begin{gathered}
x(0)=z_{0}, x(T) \geq z_{1}, \\
\sum_{t=0}^{T-1} w_{t}(y(t)) \geq U\left(\left\{w_{t}\right\}_{t=0}^{T-1}, z_{0}, z_{1}\right)-M_{0},
\end{gathered}
$$

the following inequality holds:

$$
\operatorname{Card}(\{t \in\{0, \ldots, T-1\}: \max \{\|x(t)-\widehat{x}\|,\|y(t)-\widehat{x}\|\}>\varepsilon\}) \leq L .
$$

Theorem 4.14. Suppose that for each good program $\{u(t), v(t)\}_{t=0}^{\infty}$,

$$
\lim _{t \rightarrow \infty}(u(t), v(t))=(\widehat{x}, \widehat{x}) .
$$

Let $M>\max \left\{\left(a_{i} d\right)^{-1}: i=1, \ldots, n\right\}, M_{0}>0$ and $\varepsilon>0$. Then there exist a natural number $L$ and a positive number $\tilde{\gamma}$ such that for each integer $T>L$, each $z_{0} \in R_{+}^{n}$ satisfying $z_{0} \leq M e$, each finite sequence of functions $w_{i}: R_{+}^{n} \rightarrow R^{1}, i=0, \ldots, T-1$ which are bounded on bounded subsets of $R_{+}^{n}$ and such that

$$
\left\|w_{i}-w(b(\cdot))\right\|_{M} \leq \tilde{\gamma}
$$

for each $i \in\{0, \ldots, T-1\}$ and each program $\left(\{x(t)\}_{t=0}^{T},\{y(t)\}_{t=0}^{T-1}\right)$ which satisfies

$$
\begin{gathered}
x(0)=z_{0}, \\
\sum_{t=0}^{T-1} w_{t}(y(t)) \geq U\left(\left\{w_{t}\right\}_{t=0}^{T-1}, z_{0}\right)-M_{0},
\end{gathered}
$$

the following inequality holds:

$$
\operatorname{Card}(\{t \in\{0, \ldots, T-1\}: \max \{\|x(t)-\widehat{x}\|,\|y(t)-\widehat{x}\|\}>\varepsilon\}) \leq L .
$$

\section{The Robinson-SOLOW-SRINIVASAN MODEL WITH A NONCONCAVE UTILITY FUNCTION}

In this section we consider infinite horizon optimal control problems related to the RobinsonSolow-Srinivasan model with a nonconcave utility function. In particular, we show the existence of good programs and optimal programs using different optimality criterions.

Let $a=\left(a_{1}, \ldots, a_{n}\right)>>0, b=\left(b_{1}, \ldots, b_{n}\right)>>0$, and $d \in(0,1]$.

Recall that a sequence $\{x(t), y(t)\}_{t=0}^{\infty}$ is called a program if, for each integer $t \geq 0$,

$$
\begin{gathered}
(x(t), y(t)) \in R_{+}^{n} \times R_{+}^{n}, x(t+1) \geq(1-d) x(t), \\
0 \leq y(t) \leq x(t), a(x(t+1)-(1-d) x(t))+e y(t) \leq 1 .
\end{gathered}
$$


Let $T_{1}, T_{2}$ be integers such that $0 \leq T_{1}<T_{2}$. A pair of sequences

$$
\left(\{x(t)\}_{t=T_{1}}^{T_{2}},\{y(t)\}_{t=T_{1}}^{T_{2}-1}\right)
$$

is called a program if $x\left(T_{2}\right) \in R_{+}^{n}$ and for each integer $t$ satisfying $T_{1} \leq t<T_{2}$ relations (5.1) hold.

Let $w:[0, \infty) \rightarrow[0, \infty)$ be a continuous strictly increasing function which represents the preferences of the planner.

For every point $x_{0} \in R_{+}^{n}$ and every natural number $T$, set

$$
U\left(x_{0}, T\right)=\sup \left\{\sum_{t=0}^{T-1} w(b y(t)):\left(\{x(t)\}_{t=0}^{T},\{y(t)\}_{t=0}^{T-1}\right)\right.
$$

is a program such that $\left.x(0)=x_{0}\right\}$.

In the sequel, we assume that supremum of empty set is $-\infty$.

Let $x_{0}, \tilde{x}_{0} \in R_{+}^{n}$ and let $T$ be a natural number. Set

$$
U\left(x_{0}, \tilde{x}_{0}, T\right)=\sup \left\{\sum_{t=0}^{T-1} w(b y(t)):\left(\{x(t)\}_{t=0}^{T},\{y(t)\}_{t=0}^{T-1}\right)\right.
$$

is a program such that $\left.x(0)=x_{0}, x(T) \geq \tilde{x}_{0}\right\}$.

The next proposition follows immediately from the continuity of $w$.

Proposition 5.1. For every point $x_{0} \in R_{+}^{n}$ and every integer $T>0$, there exists a program $\left(\{x(t)\}_{t=0}^{T},\{y(t)\}_{t=0}^{T-1}\right)$ such that $x(0)=x_{0}$ and

$$
\sum_{t=0}^{T-1} w(b y(t))=U\left(x_{0}, T\right)
$$

Set

$$
\Omega=\left\{\left(x, x^{\prime}\right) \in R_{+}^{n} \times R_{+}^{n}: x^{\prime} \geq(1-d) x \text { and } a\left(x^{\prime}-(1-d) x\right) \leq 1\right\} .
$$

We have a correspondence $\Lambda: \Omega \rightarrow R_{+}^{n}$ given by

$$
\begin{gathered}
\Lambda\left(x, x^{\prime}\right)=\left\{y \in R_{+}^{n}: 0 \leq y \leq x\right. \text { and } \\
\left.e y \leq 1-a\left(x^{\prime}-(1-d) x\right)\right\},\left(x, x^{\prime}\right) \in \Omega .
\end{gathered}
$$

Let $M_{0}$ be a positive number and let $T \geq 1$ be an integer. Set

$$
\begin{gathered}
\widehat{U}\left(M_{0}, T\right)=\sup \left\{\sum_{t=0}^{T-1} w(b y(t)):\right. \\
\left.\left(\{x(t)\}_{t=0}^{T},\{y(t)\}_{t=0}^{T-1}\right) \text { is a program such that } x(0) \leq M_{0} e\right\} .
\end{gathered}
$$

It is clear that $\widehat{U}\left(M_{0}, T\right)$ is finite. The next proposition follows immediately from the continuity of $w$.

Proposition 5.2. For every positive number $M_{0}$ and every integer $T \geq 1$, there exists a program $\left(\{x(t)\}_{t=0}^{T},\{y(t)\}_{t=0}^{T-1}\right)$ such that $x(0) \leq M_{0} e$ and $\sum_{t=0}^{T-1} w(b y(t))=\widehat{U}\left(M_{0}, T\right)$. 
In this section we show the existence of a positive constant $\mu$ such that the following properties hold:

(a) For each program $\{x(t), y(t)\}_{t=0}^{\infty}$, either the sequence $\left\{\sum_{t=0}^{T-1}[w(b y(t))-\mu]\right\}_{T=1}^{\infty}$ is bounded or

$$
\lim _{T \rightarrow \infty} \sum_{t=0}^{T-1}[w(b y(t))-\mu]=-\infty
$$

(b) for each $x_{0} \in R_{+}^{n}$, there exists a program $\{x(t), y(t)\}_{t=0}^{\infty}$ such that $x(0)=x_{0}$ and that the sequence $\left\{\sum_{t=0}^{T-1}[w(b y(t))-\mu]\right\}_{T=1}^{\infty}$ is bounded.

For any $\left(x, x^{\prime}\right) \in \Omega$, define

$$
u\left(x, x^{\prime}\right)=\max \left\{w(b y): y \in \Lambda\left(x, x^{\prime}\right)\right\} .
$$

In this section we state several results obtained in [60].

Our first result allows us to define the constant $\mu$.

Theorem 5.1. Let $M_{1}, M_{2}>\max \left\{\left(d a_{i}\right)^{-1}: i=1, \ldots, n\right\}$. Then there exist finite limits

$$
\lim _{p \rightarrow \infty} \widehat{U}\left(M_{i}, p\right) / p, i=1,2
$$

and

$$
\lim _{p \rightarrow \infty} \widehat{U}\left(M_{1}, p\right) / p=\lim _{p \rightarrow \infty} \widehat{U}\left(M_{2}, p\right) / p
$$

Define

$$
\mu=\lim _{p \rightarrow \infty} \widehat{U}(M, p) / p
$$

where $M>\max \left\{\left(d a_{i}\right)^{-1}: i=1, \ldots, n\right\}$. Note that $\mu$ is well defined and does not depend on $M$.

Theorem 5.2. Let $M_{0}>\max \left\{\left(d a_{i}\right)^{-1}: i=1, \ldots, n\right\}$. Then there exists $M>0$ such that

$$
\left|\widehat{U}\left(M_{0}, p\right)-p \mu\right| \leq M \text { for all integers } p \geq 1 \text {. }
$$

Corollary 5.1. Let $M_{0}>\max \left\{\left(d a_{i}\right)^{-1}: i=1, \ldots, n\right\}$. Then there exists a positive number $M$ such that, for each program $\{x(t), y(t)\}_{t=0}^{\infty}$ satisfying $x(0) \leq M_{0}$ e and each integer $T \geq 1$,

$$
\sum_{t=0}^{T-1}[w(b y(t))-\mu] \leq M .
$$

Proposition 5.3. Let $\{x(t), y(t)\}_{t=0}^{\infty}$ be a program. Then either the sequence $\left\{\sum_{t=0}^{T-1}[w(b y(t))-\right.$ $\mu]\}_{T=1}^{\infty}$ is bounded or

$$
\lim _{T \rightarrow \infty} \sum_{t=0}^{T-1}[w(b y(t))-\mu]=-\infty .
$$

Recall that a program $\{x(t), y(t)\}_{t=0}^{\infty}$ is called good if there exists $M \in R^{1}$ such that

$$
\sum_{t=0}^{T}(w(y(t))-\mu) \geq M \text { for all } T \geq 0
$$

and that it is called bad if

$$
\lim _{T \rightarrow \infty} \sum_{t=0}^{T}(w(y(t))-\mu)=-\infty .
$$

By Proposition 5.3 any program that is not good is bad. 
Set

$$
\begin{gathered}
x(t)=\left(2 n d \max \left\{a_{i}: i=1, \ldots, n\right\}\right)^{-1} e, \\
y(t)=\min \left\{(2 n)^{-1},\left(2 n d \max \left\{a_{i}: i=1, \ldots, n\right\}\right)^{-1}\right\} e \text { for all integers } t \geq 0 .
\end{gathered}
$$

It is easy to see that $\{x(t), y(t)\}_{t=0}^{\infty}$ is a program. By Corollary 5.1, we have

$$
\mu \geq \lim _{T \rightarrow \infty} T^{-1} \sum_{t=0}^{T-1} w(b y(t))>w(0) .
$$

Thus

$$
\mu>w(0)
$$

Theorem 5.3. Let $M_{0}>\max \left\{\left(d a_{i}\right)^{-1}: i=1, \ldots, n\right\}$. Then there exists a positive number $M$ such that, for every $x_{0} \in R_{+}^{n}$, which satisfies $x_{0} \leq M_{0}$ e, there exists a program $\{x(t), y(t)\}_{t=0}^{\infty}$ such that $x(0)=x_{0}$, for every integer $T_{1} \geq 0$ and every natural number $T_{2}>T_{1}$,

$$
\left|\sum_{t=T_{1}}^{T_{2}-1} w(b y(t))-\mu\left(T_{2}-T_{1}\right)\right| \leq M
$$

and that, for every natural number $T$,

$$
\sum_{t=0}^{T-1} w(b y(t))=U(x(0), x(T), T) .
$$

Theorem 5.3 establishes that for every initial state $x_{0} \geq 0$ there exists a good program $\{x(t)$, $y(t)\}_{t=0}^{\infty}$ such that $x(0)=x_{0}$. In addition, this program satisfies (5.2) for every natural number $T$. This leads us to the following definition.

A program $\{x(t), y(t)\}_{t=0}^{\infty}$ is called weakly maximal if equality (5.2) holds for every natural number $T$.

The next result establishes a relation between good programs and weakly maximal programs.

Theorem 5.4. Let $\{x(t), y(t)\}_{t=0}^{\infty}$ be a weakly maximal program such that $\lim _{\sup _{t \rightarrow \infty}} b y(t)>0$. Then the program $\{x(t), y(t)\}_{t=0}^{\infty}$ is good.

A program $\left\{x^{*}(t), y^{*}(t)\right\}_{t=0}^{\infty}$ is called weakly agreeable if, for every nonnegative integer $t$,

$$
u\left(x^{*}(t), x^{*}(t+1)\right)=w\left(b y^{*}(t)\right)
$$

and if, for every integer $T_{0} \geq 1$ and every positive number $\varepsilon$, there exists a natural number $T_{\varepsilon}>T_{0}$ such that, for every program $\left(\{x(t)\}_{t=0}^{T_{\varepsilon}},\{y(t)\}_{t=0}^{T_{\varepsilon}-1}\right)$ which satisfies $x(0)=x^{*}(0)$, there exists a program $\left(\left\{x^{\prime}(t)\right\}_{t=0}^{T_{\varepsilon}},\left\{y^{\prime}(t)\right\}_{t=0}^{T_{\varepsilon}-1}\right)$ such that

$$
\begin{gathered}
x^{\prime}(0)=x(0), x^{\prime}(t)=x^{*}(t), t=0, \ldots, T_{0}, \\
\sum_{t=0}^{T_{\varepsilon}-1} w\left(b y^{\prime}(t)\right) \geq \sum_{t=0}^{T_{\varepsilon}-1} w(b y(t))-\varepsilon .
\end{gathered}
$$

The notion of weakly agreeable programs is a weakened version of the notion of agreeable programs which is well-known in the literature $[15,16,17]$.

The following three results were obtained in [72].

Theorem 5.5. Any weakly agreeable program is good.

Theorem 5.6. Any weakly agreeable program is weakly maximal. 
Theorem 5.7. A program $\left\{x^{*}(t), y^{*}(t)\right\}_{t=0}^{\infty}$ is weakly agreeable if and only if there exist a strictly increasing sequence of natural numbers $\left\{S_{k}\right\}_{k=1}^{\infty}$ and a sequence of programs

$$
\left(\left\{x^{(k)}(t)\right\}_{t=0}^{S_{k}},\left\{y^{(k)}(t)\right\}_{t=0}^{S_{k}-1}\right), k=1,2, \ldots
$$

such that

$$
\begin{gathered}
x^{(k)}(0)=x^{*}(0), k=1,2, \ldots \\
U\left(x^{*}(0), S_{k}\right)-\sum_{t=0}^{S_{k}-1} w\left(b y^{(k)}(t)\right) \rightarrow 0 \text { as } k \rightarrow \infty
\end{gathered}
$$

and that, for all integers $t \geq 0$,

$$
x^{*}(t)=\lim _{k \rightarrow \infty} x^{(k)}(t), y^{*}(t)=\lim _{k \rightarrow \infty} y^{(k)}(t) .
$$

The following three theorems were obtained in [66].

We begin with the result which establishes the continuity of the function $U(\cdot, \cdot, T)$.

Theorem 5.8. Let $T>0$ be an integer, $x_{0}, \tilde{x}_{0} \in R_{+}^{n}, U\left(x_{0}, \tilde{x}_{0}, T\right)>0$ and let $d<1$. Then the function $(y, z) \rightarrow U(y, z, T), y, z \in R_{+}^{n}$ is continuous at $\left(x_{0}, \tilde{x}_{0}\right)$.

Theorem 5.9. Let $M_{0}>\max \left\{\left(d a_{i}\right)^{-1}: i=1, \ldots, n\right\}$. Then there exists $M_{1}>0$ such that for each good weakly maximal program $\{x(t), y(t)\}_{t=0}^{\infty}$ satisfying $x(0) \leq M_{0} e$ and each pair of integers $S_{1} \geq 0$ and $S_{2}>S_{1}$,

$$
\left|\sum_{t=S_{1}}^{S_{2}-1} w(b y(t))-\mu\left(S_{2}-S_{1}\right)\right| \leq M_{1} .
$$

By definition for any good program $\{x(t), y(t)\}_{t=0}^{\infty}$, there is a constant $M_{1}>0$ such that the inequality above holds. In view of Theorem 5.9, the constant $M_{1}$ depends only on the constant $M_{0}$ and the inequality above holds for all programs $\{x(t), y(t)\}_{t=0}^{\infty}$ satisfying $x(0) \leq M_{0} e$.

Theorem 5.10. Let $\left\{x^{(k)}(t), y^{(k)}(t)\right\}_{t=0}^{\infty}, k=1,2, \ldots$ be good weakly maximal programs. Assume that, for any integer $t \geq 0$, there exists $x(t)=\lim _{k \rightarrow \infty} x^{(k)}(t), y(t)=\lim _{k \rightarrow \infty} y_{t}^{(k)}$. Then $\{x(t), y(t)\}_{t=0}^{\infty}$ is a good weakly maximal program.

Now we consider the RSS model with discounting. For every nonnegative integer $t$ let $w_{t}$ : $[0, \infty) \rightarrow[0, \infty)$ be a continuous increasing function which represents the preferences of the planner at moment of time $t$. We suppose that the following assumption holds.

Assumption A: For every nonnegative integer $t \geq 0, w_{t}(0)=0$, and for every positive number $M$,

$$
\lim _{t \rightarrow \infty} w_{t}(M)=0 .
$$

For every point $x_{0} \in R_{+}^{n}$ and every natural number $T$, define

$$
U\left(x_{0}, T\right)=\sup \left\{\sum_{t=0}^{T-1} w_{t}(b y(t)):\left(\{x(t)\}_{t=0}^{T},\{y(t)\}_{t=0}^{T-1}\right)\right.
$$

is a program from such that $\left.x(0)=x_{0}\right\}$.

The next proposition follows immediately from the continuity of $w_{t}, t=0,1, \ldots$. 
Proposition 5.4. For every $x_{0} \in R_{+}^{n}$ and every integer $T \geq 1$, there exists a program $\left(\{x(t)\}_{t=0}^{T}\right.$, $\left.\{y(t)\}_{t=0}^{T-1}\right)$, which satisfies $x(0)=x_{0}$ and

$$
\sum_{t=0}^{T-1} w_{t}(b y(t))=U\left(x_{0}, T\right) .
$$

The following theorem was obtained in [31].

Theorem 5.11. For every $z \in R_{+}^{n}$ there exists a program $\left\{x_{z}(t), y_{z}(t)\right\}_{t=0}^{\infty}$ such that $x_{z}(0)=z$ and the following property holds: For every pair of positive numbers $M_{0}, \delta$, there exists an integer $L^{(\delta)} \geq 1$ such that, for every natural number $S \geq L^{(\delta)}$ and every $z \in R_{+}^{n}$, which satisfies $z \leq M_{0} e$,

$$
\sum_{t=0}^{S-1} w_{t}\left(b y_{z}(t)\right) \geq U(z, S)-\delta .
$$

Corollary 5.2. Let $z \in R_{+}^{n}$ and let a program $\left\{x_{z}(t), y_{z}(t)\right\}_{t=0}^{\infty}$ be as guaranteed by Theorem 5.11. Then, for every program $\{x(t), y(t)\}_{t=0}^{\infty}$ satisfying $x(0)=z$, the inequality

$$
\liminf _{T \rightarrow \infty}\left[\sum_{t=0}^{T-1} w_{t}\left(b y_{z}(t)\right)-\sum_{t=0}^{T-1} w_{t}(b y(t))\right] \geq 0
$$

holds.

Example 5.1. Let $w:[0, \infty) \rightarrow[0, \infty)$ be a continuous increasing function, $w(0)=0,\left\{\rho_{t}\right\}_{t=0}^{\infty} \subset$ $(0,1), \lim _{t \rightarrow \infty} \rho_{t}=0$, and $w_{t}=\rho_{t} w, t=0,1, \ldots$ Then Assumption A holds. Assume that $\sum_{t=0}^{\infty} \rho_{t}=\infty$ and that $w(s)>0$ for every positive number $s$. Let $z \in R_{+}^{n}$ be given. Set $x(0)=$ $z, y(0)=0$, for every natural number $t$,

$$
y(t)=(2 n)^{-1} \min \left\{1, a_{1}^{-1}, \ldots, a_{i}^{-1}, \ldots, a_{n}^{-1}\right\} e,
$$

for every nonnegative integer $t$,

$$
x(t+1)=(1-d) x(t)+(2 n)^{-1}\left(a_{1}^{-1}, \ldots, a_{n}^{-1}\right) .
$$

Evidently, $\{x(t), y(t)\}_{t=0}^{\infty}$ is a program and, for every natural number $T$,

$$
\begin{aligned}
\sum_{t=0}^{T} w_{t}(b y(t)) & =\sum_{t=1}^{T} \rho_{t} w\left(b e(2 n)^{-1} \min \left\{1, a_{1}^{-1}, \ldots, a_{n}^{-1}\right\}\right) \\
& =\left(\sum_{t=1}^{T} \rho_{t}\right) w\left(b e(2 n)^{-1} \min \left\{1, a_{1}^{-1}, \ldots, a_{n}^{-1}\right\}\right) \rightarrow \infty \text { as } T \rightarrow \infty .
\end{aligned}
$$

This implies that $U(z, T) \rightarrow \infty$ as $T \rightarrow \infty$.

\section{NOAUTONOMOUS INFINITE HORIZON OPTIMAL CONTROL PROBLEMS}

In this section we study infinite horizon optimal control problems with noautonomous optimality criterions. The utility functions, which determine the optimality criterion, are nonconcave. The class of models contains, as a particular case, the Robinson-Solow-Srinivasan model. We show the existence of good programs and optimal programs.

For every $x \in R^{n}$, we denote by $\|x\|_{2}$ its Euclidean norm in $R^{n}$. We assume that $\|\cdot\|$ is a norm in $R^{n}$. For every mapping $a: X \rightarrow 2^{Y} \backslash\{\emptyset\}$, where $X, Y$ are nonempty sets, we set

$$
\operatorname{graph}(a)=\{(x, y) \in X \times Y: y \in a(x)\} .
$$


Let $K$ be a nonempty compact subset of $R^{n}$. Denote by $\mathscr{P}(K)$ the set of all nonempty closed subsets of $K$. For every pair of nonempty sets $A, B \subset R^{n}$, define

$$
H(A, B)=\sup \left\{\sup _{x \in A} \inf _{y \in B}\|x-y\|, \sup _{y \in B} \inf _{x \in A}\|x-y\|\right\} .
$$

For every nonnegative integer $t$, let $a_{t}: K \rightarrow \mathscr{P}(K)$ be such that graph $\left(a_{t}\right)$ is a closed subset of $R^{n} \times R^{n}$. Assume that there exists a number $\kappa \in(0,1)$ such that, for every pair of points $x, y \in K$ and every nonnegative integer $t$,

$$
H\left(a_{t}(x), a_{t}(y)\right) \leq \kappa\|x-y\|
$$

and that, for every nonnegative integer $t$, the upper semicontinuous function

$$
u_{t}:\left\{\left(x, x^{\prime}\right) \in K \times K, x^{\prime} \in a_{t}(x)\right\} \rightarrow[0, \infty)
$$

satisfies

$$
\sup \left\{\sup \left\{u_{t}\left(x, x^{\prime}\right):\left(x, x^{\prime}\right) \in \operatorname{graph}\left(a_{t}\right)\right\}: t=0,1, \ldots\right\}<\infty .
$$

A sequence $\{x(t)\}_{t=0}^{\infty} \subset K$ is called a program if $x(t+1) \in a_{t}(x(t))$ for every nonnegative integer $t$.

Let $T_{1}, T_{2}$ be integers such that $T_{1}<T_{2}$. A sequence $\{x(t)\}_{t=T_{1}}^{T_{2}} \subset K$ is called a program if $x(t+1) \in a_{t}(x(t))$ for every integer $t$ satisfying $T_{1} \leq t<T_{2}$.

We suppose that the following assumptions hold:

(A1) for every positive number $\delta$, there exists a positive number $\lambda$ such that if an integer $t \geq 0$ and if $\left(x, x^{\prime}\right) \in \operatorname{graph}\left(a_{t}\right)$ satisfies $u_{t}\left(x, x^{\prime}\right) \geq \delta$, then there exists $z \in a_{t}(x)$ satisfying $z \geq x^{\prime}+\lambda e$;

(A2) there exist a program $\{\widehat{x}(t)\}_{t=0}^{\infty}$ and a positive number $\widehat{\Delta}$ such that $u_{t}(\widehat{x}(t), \widehat{x}(t+1)) \geq \widehat{\Delta}$ for every nonnegative integer $t$;

(A3) for every nonnegative integer $t$, every $(x, y) \in \operatorname{graph}\left(a_{t}\right)$ and every $\tilde{x} \in K$, which satisfies $\tilde{x} \geq x$, there exists $\tilde{y} \in a_{t}(\tilde{x})$ for which

$$
\tilde{y} \geq y, u_{t}(\tilde{x}, \tilde{y}) \geq u_{t}(x, y) .
$$

In the sequel, we assume that supremum of empty set is $-\infty$.

For every point $x_{0} \in K$ and every natural number $T$, define

$$
\begin{gathered}
U\left(x_{0}, T\right)=\sup \left\{\sum_{t=0}^{T-1} u_{t}(x(t), x(t+1)):\right. \\
\left.\{x(t)\}_{t=0}^{T-1} \text { is a program and } x(0)=x_{0}\right\} .
\end{gathered}
$$

Let $x_{0}, \tilde{x}_{0} \in K$ and let $T \geq 1$ be an integer. Define

$$
\begin{gathered}
U\left(x_{0}, \tilde{x}_{0}, T\right)=\sup \left\{\sum_{t=0}^{T-1} u_{t}(x(t), x(t+1)):\{x(t)\}_{t=0}^{T-1}\right. \text { is a program such that } \\
\left.x(0)=x_{0}, x(T) \geq \tilde{x}_{0}\right\} .
\end{gathered}
$$

Let $T \geq 1$ be an integer. Define

$$
\widehat{U}(T)=\sup \left\{\sum_{t=0}^{T-1} u_{t}(x(t), x(t+1)):\{x(t)\}_{t=0}^{T-1} \text { is a program }\right\} .
$$

The results presented in this section were obtained in [63].

Upper semicontinuity of $u_{t}, t=0,1, \ldots$ implies the following two propositions. 
Proposition 6.1. For every $x_{0} \in K$ and every integer $T \geq 1$, there exists a program $\{x(t)\}_{t=0}^{T}$, which satisfies $x(0)=x_{0}$ and

$$
\sum_{t=0}^{T-1} u_{t}(x(t), x(t+1))=U\left(x_{0}, T\right)
$$

Proposition 6.2. For every integer $T \geq 1$ there exists a program $\{x(t)\}_{t=0}^{T}$ satisfying

$$
\sum_{t=0}^{T-1} u_{t}(x(t), x(t+1))=\widehat{U}(T) .
$$

For every $x_{0} \in K$ and every pair of integers $T_{1}<T_{2}$, define

$$
\begin{gathered}
U\left(x_{0}, T_{1}, T_{2}\right)=\sup \left\{\sum_{t=T_{1}}^{T_{2}-1} u_{t}(x(t), x(t+1)):\right. \\
\left.\{x(t)\}_{t=T_{1}}^{T_{2}-1} \text { is a program and } x\left(T_{1}\right)=x_{0}\right\} .
\end{gathered}
$$

Upper semicontinuity of $u_{t}, t=0,1, \ldots$ implies the following result.

Proposition 6.3. For every $x_{0} \in K$ and every pair of integers $T_{1}<T_{2}$ there exists a program $\{x(t)\}_{t=T_{1}}^{T_{2}}$ such that $x\left(T_{1}\right)=x_{0}$ and

$$
\sum_{t=T_{1}}^{T_{2}-1} u_{t}(x(t), x(t+1))=U\left(x_{0}, T_{1}, T_{2}\right)
$$

Let $x_{0}, \tilde{x}_{0} \in K$ and let $T_{1}<T_{2}$ be integers. Define

$$
\begin{gathered}
U\left(x_{0}, \tilde{x}_{0}, T_{1}, T_{2}\right)=\sup \left\{\sum_{t=T_{1}}^{T_{2}-1} u_{t}(x(t), x(t+1)):\{x(t)\}_{t=T_{1}}^{T_{2}}\right. \text { is a program and } \\
\left.x\left(T_{1}\right)=x_{0}, x\left(T_{2}\right) \geq \tilde{x}_{0}\right\} .
\end{gathered}
$$

Let $T_{1}, T_{2}$ be integers such that $T_{1}<T_{2}$. Define

$$
\widehat{U}\left(T_{1}, T_{2}\right)=\sup \left\{\sum_{t=T_{1}}^{T_{2}-1} u_{t}(x(t), x(t+1)):\{x(t)\}_{t=T_{1}}^{T_{2}} \text { is a program }\right\} .
$$

The following theorem is the main result of this section.

Theorem 6.1. There exists a positive number $M$ such that for every $x_{0} \in K$ there exists a pro$\operatorname{gram}\{\bar{x}(t)\}_{t=0}^{\infty}$ such that $\bar{x}(0)=x_{0}$ and that for every pair of nonnegative integers $T_{1}, T_{2}$ satisfying $T_{1}<T_{2}$, the inequality

$$
\left|\sum_{t=T_{1}}^{T_{2}-1} u_{t}(\bar{x}(t), \bar{x}(t+1))-\widehat{U}\left(T_{1}, T_{2}\right)\right| \leq M
$$

holds. Moreover, for every natural number $T$,

$$
\sum_{t=0}^{T-1} u_{t}(\bar{x}(t), \bar{x}(t+1))=\tilde{U}(\bar{x}(0), \bar{x}(T), 0, T),
$$

if the following properties hold: 
for every nonnegative integer $t$ and every $\left(z, z^{\prime}\right) \in \operatorname{graph}\left(a_{t}\right)$ satisfying $u_{t}\left(z, z^{\prime}\right)>0$, the function $u_{t}$ is continuous at the point $\left(z, z^{\prime}\right)$;

for every nonnegative integer $t$ and each $z, z_{1}, z_{2}, z_{3} \in K$, which satisfy $z_{1} \leq z_{2} \leq z_{3}$ and $z_{i} \in a_{t}(z), i=1,3$, the inclusion $z_{2} \in a_{t}(z)$ is valid.

The program $\{\bar{x}(t)\}_{t=0}^{\infty}$ whose existence is guaranteed by Theorem 6.1 in infinite horizon optimal control is considered as an (approximately) optimal program.

Theorem 6.2. Assume that $\{x(t)\}_{t=0}^{\infty}$ is a program. There exists a positive number $M_{0}$ such that, for every natural number $T$,

$$
\sum_{t=0}^{T-1} u_{t}(x(t), x(t+1)) \geq U(0, T, x(0), x(T))-M_{0}
$$

and that

$$
\limsup _{t \rightarrow \infty} u_{t}(x(t), x(t+1))>0 .
$$

Then there exists positive number $M_{1}$ such that, for every pair of integers $T_{1} \geq 0$ satisfying $T_{2}>T_{1}$, the inequality

$$
\left|\sum_{t=T_{1}}^{T_{2}-1} u_{t}(x(t), x(t+1))-\widehat{U}\left(T_{1}, T_{2}\right)\right| \leq M_{1}
$$

holds.

Proposition 6.4. Let $x_{0} \in K$ and let a program $\{\bar{x}(t)\}_{t=0}^{\infty}$ be as guaranteed by Theorem 6.1. Assume that $\{x(t)\}_{t=0}^{\infty}$ is a program. Then either the sequence

$$
\left\{\sum_{t=0}^{T-1} u_{t}(x(t), x(t+1))-\sum_{t=0}^{T-1} u_{t}(\bar{x}(t), \bar{x}(t+1))\right\}_{T=1}^{\infty}
$$

is bounded or

$$
\sum_{t=0}^{T-1} u_{t}(x(t), x(t+1))-\sum_{t=0}^{T-1} u_{t}(\bar{x}(t), \bar{x}(t+1)) \rightarrow-\infty \text { as } T \rightarrow \infty .
$$

Now assume that $u_{t}=u_{0}$ and $a_{t}=a_{0}, t=0,1, \ldots$ Let a positive number $M$ be as guaranteed by Theorem 6.1 and set $u=u_{0}, a=a_{0}$.

Theorem 6.3. There exists $\mu=\lim _{p \rightarrow \infty} \widehat{U}(0, p) / p$ and

$$
\left|p^{-1} \widehat{U}(0, p)-\mu\right| \leq 2 M / p \text { for all natural numbers } p .
$$

\section{TURNPIKE RESULTS FOR THE NONSTATIONARY ROBINSON-SOLOW-SRINIVASAN}

MODEL

In this section we discuss turnpike results for a class of discrete-time optimal control problems. These control problems arise in economic dynamics and describe the nonstationary onedimensional Robinson-Solow-Srinivasan model. We study the structure of approximate solutions which is independent of the length of the interval, for all sufficiently large intervals. The results of this chapter were obtained in [64]. 
Denote by $\operatorname{Card}(E)$ the cardinality of a set $E$. Let $R^{1}\left(R_{+}^{1}\right)$ be the set of real (nonnegative) numbers. For each mapping $a: X \rightarrow 2^{Y} \backslash\{\emptyset\}$, where $X, Y$ are nonempty sets, put $\operatorname{graph}(a)=$ $\{(x, y) \in X \times Y: y \in a(x)\}$. For each integer $t \geq 0$, let

$$
\alpha_{t}>0, d_{t} \in(0,1]
$$

and, for each integer $t \geq 0$, let $w_{t}:[0, \infty) \rightarrow[0, \infty)$ be a strictly increasing continuous function such that

$$
w_{t}(0)=0 \text { and } \inf \left\{w_{t}(z): \text { an integer } t \geq 0\right\}>0 \text { for all } z>0 .
$$

We suppose that the following assumption holds:

(A1) for each $\varepsilon>0$, there exists $\delta>0$ such that $w_{t}(\delta) \leq \varepsilon$ for each integer $t \geq 0$.

We now give a formal description of the model.

Let $t \geq 0$ be an integer. For each $x \in R_{+}^{1}$, set

$$
a_{t}(x)=\left\{y \in R_{+}^{1}: y \geq\left(1-d_{t}\right) x \text { and } \alpha_{t}\left(y-\left(1-d_{t}\right) x\right) \leq 1\right\} .
$$

It is clear that, for each $x \in R_{+}^{1}$,

$$
a_{t}(x)=\left[\left(1-d_{t}\right) x, \alpha_{t}^{-1}+\left(1-d_{t}\right) x\right]
$$

and that $\operatorname{graph}\left(a_{t}\right)$ is a closed subset of $R_{+}^{1} \times R_{+}^{1}$. Suppose that

$$
\begin{gathered}
\inf \left\{d_{t}: t=0,1, \ldots\right\}>0, \\
\inf \left\{\alpha_{t}: t=0,1, \ldots\right\}>0, \\
\sup \left\{\alpha_{t}: t=0,1, \ldots\right\}<\infty, \\
\sup \left\{w_{t}(M): t=0,1, \ldots\right\}<\infty \text { for each } M>0 .
\end{gathered}
$$

The constraint mappings $a_{t}, t=0,1, \ldots$ have already been defined. Now we define the cost functions $u_{t}, t=0,1, \ldots$. For each integer $t \geq 0$ and each $\left(x, x^{\prime}\right) \in \operatorname{graph}\left(a_{t}\right)$, set

$$
u_{t}\left(x, x^{\prime}\right)=\sup \left\{w_{t}(y): 0 \leq y \leq x \text { and } y+\alpha_{t}\left(x^{\prime}-\left(1-d_{t}\right) x\right) \leq 1\right\} .
$$

Clearly, for each integer $t \geq 0$ and each $\left(x, x^{\prime}\right) \in \operatorname{graph}\left(a_{t}\right)$,

$$
u_{t}\left(x, x^{\prime}\right)=w_{t}\left(\min \left\{x, 1-\alpha_{t}\left(x^{\prime}-\left(1-d_{t}\right) x\right)\right\}\right) .
$$

Choose $\alpha^{*}, \alpha_{*}, d_{*}>0$ such that

$$
\alpha_{*}<\alpha_{t}<\alpha^{*}, d_{*}<d_{t} \text { for all integers } t \geq 0 .
$$

Clearly, for each integer $t \geq 0$, the function $u_{t}: \operatorname{graph}\left(a_{t}\right) \rightarrow[0, \infty)$ is upper semicontinuous.

A sequence $\{x(t)\}_{t=0}^{\infty} \subset R_{+}^{1}$ is called a program if $x(t+1) \in a_{t}(x(t))$ for all integers $t \geq 0$. Let $T_{1}, T_{2}$ be integers such that $T_{1}<T_{2}$. A sequence $\{x(t)\}_{t=T_{1}}^{T_{2}} \subset R_{+}^{1}$ is called a program if $x(t+1) \in a_{t}(x(t))$ for all integers $t$ satisfying $T_{1} \leq t<T_{2}$. In the sequel, we assume that the supremum over an empty set is $-\infty$.

For each $x_{0} \in R_{+}^{1}$ and each pair of integers $T_{1}<T_{2}$, set

$$
U\left(x_{0}, T_{1}, T_{2}\right)=\sup \left\{\sum_{t=T_{1}}^{T_{2}-1} u_{t}(x(t), x(t+1)):\{x(t)\}_{t=T_{1}}^{T_{2}}\right.
$$

is a program and $\left.x\left(T_{1}\right)=x_{0}\right\}$. 
Let $x_{0}, \tilde{x}_{0} \in R_{+}^{1}$ and let $T_{1}<T_{2}$ be integers. Set

$$
U\left(x_{0}, \tilde{x}_{0}, T_{1}, T_{2}\right)=\sup \left\{\sum_{t=T_{1}}^{T_{2}-1} u_{t}(x(t), x(t+1)):\{x(t)\}_{t=T_{1}}^{T_{2}}\right.
$$

is a program such that $\left.x\left(T_{1}\right)=x_{0}, x\left(T_{2}\right) \geq \tilde{x}_{0}\right\}$.

Let $T_{1}, T_{2}$ be integers such that $T_{1}<T_{2}$. Set

$$
\begin{aligned}
& \widehat{U}_{M}\left(T_{1}, T_{2}\right)=\sup \left\{\sum_{t=T_{1}}^{T_{2}-1} u_{t}(x(t), x(t+1)):\right. \\
& \left.\{x(t)\}_{t=T_{1}}^{T_{2}} \text { is a program and } x\left(T_{1}\right) \leq M\right\} .
\end{aligned}
$$

Upper semicontinuity of $u_{t}, t=0,1, \ldots$, compactness of sets of admissible programs and the optimization theorem of Weierstrass imply the following results.

Proposition 7.1. For each $x_{0} \in R_{+}^{1}$ and each pair of integers $T_{1}<T_{2}$, there exists a program $\{x(t)\}_{t=T_{1}}^{T_{2}}$ such that $x\left(T_{1}\right)=x_{0}$ and

$$
\sum_{t=T_{1}}^{T_{2}-1} u_{t}(x(t), x(t+1))=U\left(x_{0}, T_{1}, T_{2}\right) .
$$

Proposition 7.2. For each natural number $T$ and each $M>0$ there exists a program $\{x(t)\}_{t=0}^{T}$ such that

$$
\sum_{t=0}^{T-1} u_{t}(x(t), x(t+1))=\widehat{U}_{M}(0, T)
$$

and $x(0) \leq M$.

Fix

$$
M_{*}>\left(\alpha_{*} d_{*}\right)^{-1}+1
$$

It is clear that the model considered here is a particular case of the model discussed in Section 6 with $n=1$. Therefore all the results of Section 6 can be applied.

Theorem 7.1. There exists $\bar{M}>0$ such that, for each $x_{0} \in\left[0, M_{*}\right]$, there is a program $\{\bar{x}(t)\}_{t=0}^{\infty}$ such that $\bar{x}(0)=x_{0}$, for each pair of integers $T_{1}, T_{2} \geq 0$ satisfying $T_{1}<T_{2}$,

$$
\left|\sum_{t=T_{1}}^{T_{2}-1} u_{t}(\bar{x}(t), \bar{x}(t+1))-\widehat{U}_{M_{*}}\left(T_{1}, T_{2}\right)\right| \leq \bar{M}
$$

and that, for each integer $T>0$,

$$
\sum_{t=0}^{T-1} u_{t}(\bar{x}(t), \bar{x}(t+1))=U(\bar{x}(0), \bar{x}(T), 0, T) .
$$

Theorem 7.2. Let $x_{0} \in\left[0, M_{*}\right]$ and let a program $\{\bar{x}(t)\}_{t=0}^{\infty}$ be as guaranteed by Theorem 7.1. Assume that $\{x(t)\}_{t=0}^{\infty}$ is a program. Then either the sequence $\left\{\sum_{t=0}^{T-1} u_{t}(x(t), x(t+1))-\right.$ $\left.\sum_{t=0}^{T-1} u_{t}(\bar{x}(t), \bar{x}(t+1))\right\}_{T=1}^{\infty}$ is bounded or

$$
\sum_{t=0}^{T-1} u_{t}(x(t), x(t+1))-\sum_{t=0}^{T-1} u_{t}(\bar{x}(t), \bar{x}(t+1)) \rightarrow-\infty \text { as } T \rightarrow \infty .
$$


Let $\bar{M}>0$ be as guaranteed by Theorem 7.1. Fix $x_{* 0} \in\left[0, M_{*}\right]$ and let a program $\left\{x^{*}(t)\right\}_{t=0}^{\infty}$ be as guaranteed by Theorem 7.1. Namely,

$$
\begin{aligned}
x^{*}(0) & =x_{* 0}, \\
\sum_{t=0}^{T-1} u_{t}\left(x^{*}(t), x^{*}(t+1)\right) & =U\left(x^{*}(0), x^{*}(T), 0, T\right)
\end{aligned}
$$

for each integer $T>0$ and

$$
\left|\sum_{t=T_{1}}^{T_{2}-1} u_{t}\left(x^{*}(t), x^{*}(t+1)\right)-\widehat{U}_{M_{*}}\left(T_{1}, T_{2}\right)\right| \leq \bar{M}
$$

for each pair of integers $T_{1}, T_{2}$ satisfying $0 \leq T_{1}<T_{2}$.

For each integer $t \geq 0$, set

$$
y^{*}(t)=\min \left\{x^{*}(t), 1-\alpha_{t}\left(x^{*}(t+1)-\left(1-d_{t}\right) x^{*}(t)\right)\right\} .
$$

We will show that the program $\left\{x^{*}(t)\right\}_{t=0}^{\infty}$ is the turnpike for the model.

A function $w:[0, \infty) \rightarrow R^{1}$ is called strictly concave if, for each $x, y \in[0, \infty)$ satisfying $x \neq y$ and each $\alpha \in(0,1)$,

$$
w(\alpha x+(1-\alpha) y)>\alpha w(x)+(1-\alpha) w(y) .
$$

The following two results are consequences of the optimization theorem of Weierstrass.

Proposition 7.3. Assume that $w:[0, \infty) \rightarrow[0, \infty)$ is continuous strictly concave function. Let $\varepsilon, M>0$. Then there exists $\delta_{0}>0$ such that, for each $x, y \in[0, M]$ satisfying $|x-y| \geq \varepsilon$,

$$
w\left(2^{-1}(x+y)\right)-2^{-1} w(x)-2^{-1} w(y) \geq \delta_{0} .
$$

Proposition 7.4. Assume that $w:[0, \infty) \rightarrow[0, \infty)$ is a strictly increasing continuous function, $M>0$ and $\varepsilon \in(0, M)$. Then $\inf \{w(x)-w(y): x, y \in[0, M]$ and $x \geq y+\varepsilon\}>0$.

We suppose that the following assumptions hold.

(A2) For each $\varepsilon, M>0$, there exists $\varepsilon_{0}>0$ such that, for each $x, y \in(0, M]$ satisfying $|x-y| \geq$ $\varepsilon$ and each integer $t \geq 0$,

$$
w_{t}\left(2^{-1}(x+y)\right)-2^{-1} w_{t}(x)-2^{-1} w_{t}(y) \geq \varepsilon_{0} .
$$

(A3) For each $M>0$ and each $\varepsilon \in(0, M]$, there is $\varepsilon_{1}>0$ such that, for each integer $t \geq 0$ and each $x, y \in[0, M]$ satisfying $x \geq y+\varepsilon$,

$$
w_{t}(x)-w_{t}(y) \geq \varepsilon_{1} .
$$

(A4) For each $M>0$ and each $\varepsilon>0$, there exists $\delta>0$ such that, for each integer $t \geq 0$ and each $x, y \in[0, M]$ satisfying $|x-y| \leq \delta$, the inequality $\left|w_{t}(x)-w_{t}(y)\right| \leq \varepsilon$ holds.

Note that (A2) is an assumption of uniform concavity of the functions $w_{t}, t=0,1, \ldots$, (A3) is an assumption of uniform strict monotonicity of the functions $w_{t}, t=0,1, \ldots$ and (A4) is an assumption of uniform equicontinuity of the functions $w_{t}, t=0,1, \ldots$

We assume that

$$
d^{*}:=\sup \left\{d_{t}: t=0,1, \ldots\right\}<1 .
$$

The following theorems describe the structure of optimal program of the model. 
Theorem 7.3. Let $M>0$ and $\varepsilon>0$. Then there exists a natural number $Q$ such that, for each pair of integers $T_{1} \geq 0$ and $T_{2} \geq Q+T_{1}$ and each program $\{x(t)\}_{t=T_{1}}^{T_{2}}$, which satisfies $x\left(T_{1}\right) \leq M_{*}, \sum_{t=T_{1}}^{T_{2}-1} u_{t}(x(t), x(t+1)) \geq U\left(x\left(T_{1}\right), T_{1}, T_{2}\right)-M$, the following inequality holds:

$$
\operatorname{Card}\left(\left\{t \in\left\{T_{1}, \ldots, T_{2}\right\}:\left|x(t)-x^{*}(t)\right|>\varepsilon\right\}\right) \leq Q .
$$

Theorem 7.4. Let $M, \varepsilon>0$. Then there exist a natural number $p$ and $\delta>0$ such that, for each pair of integers $T_{1} \geq 0, T_{2} \geq 2 p+T_{1}$ and each program $\{x(t)\}_{t=T_{1}}^{T_{2}}$ satisfying $x\left(T_{1}\right) \leq M_{*}$, $\sum_{t=T_{1}}^{T_{2}-1} u_{t}(x(t), x(t+1)) \geq U\left(x\left(T_{1}\right), x\left(T_{2}\right), T_{1}, T_{2}\right)-\delta$,

$$
U\left(x\left(T_{1}\right), x\left(T_{2}\right), T_{1}, T_{2}\right) \geq U\left(x\left(T_{1}\right), T_{1}, T_{2}\right)-M,
$$

the inequality $\left|x(t)-x^{*}(t)\right| \leq \varepsilon$ holds for all integers $t \in\left[T_{1}+p, T_{2}-p\right]$.

Theorem 7.5. Let $M>0$ and $\varepsilon>0$. Then there exist a natural number $p$ and $\delta>0$ such that, for each pair of integers $T_{1} \geq 0, T_{2} \geq p+T_{1}$ and each program $\{x(t)\}_{t=T_{1}}^{T_{2}}$ which satisfies $x\left(T_{1}\right) \leq M_{*},\left|x\left(T_{1}\right)-x^{*}\left(T_{1}\right)\right| \leq \delta$,

$$
\begin{gathered}
\sum_{t=T_{1}}^{T_{2}-1} u_{t}(x(t), x(t+1)) \geq U\left(x\left(T_{1}\right), x\left(T_{2}\right), T_{1}, T_{2}\right)-\delta, \\
U\left(x\left(T_{1}\right), x\left(T_{2}\right), T_{1}, T_{2}\right) \geq U\left(x\left(T_{1}\right), T_{1}, T_{2}\right)-M,
\end{gathered}
$$

the inequality $\left|x(t)-x^{*}(t)\right| \leq \varepsilon$ holds for all integers $t \in\left[T_{1}, T_{2}-p\right]$.

Theorem 7.6. Let $\varepsilon>0$. Then there exist a natural number $p$ and $\delta>0$ such that, for each pair of integers $T_{1} \geq 0, T_{2} \geq 2 p+T_{1}$ and each program $\{x(t)\}_{t=T_{1}}^{T_{2}}$, which satisfies $x\left(T_{1}\right) \leq$ $M_{*}, \sum_{t=T_{1}}^{T_{2}-1} u_{t}(x(t), x(t+1)) \geq U\left(x\left(T_{1}\right), T_{1}, T_{2}\right)-\delta$, the inequality $\left|x(t)-x^{*}(t)\right| \leq \varepsilon$ holds for all integers $t \in\left[T_{1}+p, T_{2}-p\right]$.

Theorem 7.7. Let $\varepsilon>0$. Then there exist a natural number $p$ and $\delta>0$ such that, for each pair of integers $T_{1} \geq 0, T_{2} \geq p+T_{1}$ and each program $\{x(t)\}_{t=T_{1}}^{T_{2}}$ which satisfies $x\left(T_{1}\right) \leq M_{*}, \mid x\left(T_{1}\right)-$ $x^{*}\left(T_{1}\right) \mid \leq \delta$,

$$
\sum_{t=T_{1}}^{T_{2}-1} u_{t}(x(t), x(t+1)) \geq U\left(x\left(T_{1}\right), T_{1}, T_{2}\right)-\delta,
$$

the inequality $\left|x(t)-x^{*}(t)\right| \leq \varepsilon$ holds for all integers $t \in\left[T_{1}, T_{2}-p\right]$.

Theorems 7.6 and 7.7 easily follow from Theorems 7.4 and 7.5, respectively.

A program $\{x(t)\}_{t=0}^{\infty}$ is called good if the sequence

$$
\left\{\sum_{t=0}^{T-1} u_{t}(x(t), x(t+1))-\sum_{t=0}^{T-1} u_{t}\left(x^{*}(t), x^{*}(t+1)\right)\right\}_{T=1}^{\infty}
$$

is bounded. In view of Theorem 7.2, if the sequence $\{x(t)\}_{t=0}^{\infty}$ is not good, then

$$
\lim _{T \rightarrow \infty}\left[\sum_{t=0}^{T-1} u_{t}(x(t), x(t+1))-\sum_{t=0}^{T-1} u_{t}\left(x^{*}(t), x^{*}(t+1)\right)\right]=-\infty .
$$

Theorem 7.8. Assume that a program $\{x(t)\}_{t=0}^{\infty}$ is good. Then $x(t)-x^{*}(t) \rightarrow 0$ as $t \rightarrow \infty$. 
A program $\{x(t)\}_{t=0}^{\infty}$ is called overtaking optimal if, for each program $\left\{x^{\prime}(t)\right\}_{t=0}^{\infty}$ satisfying $x^{\prime}(0)=x(0)$,

$$
\limsup _{T \rightarrow \infty}\left[\sum_{t=0}^{T-1} u_{t}\left(x^{\prime}(t), x^{\prime}(t+1)\right)-\sum_{t=0}^{T-1} u_{t}(x(t), x(t+1))\right] \leq 0 .
$$

We have the following result.

Theorem 7.9. Let $x_{0} \in\left[0, M_{*}\right]$ and let a program $\{\bar{x}(t)\}_{t=0}^{\infty}$ be as guaranteed by Theorem 7.3. Then $\{\bar{x}(t)\}_{t=0}^{\infty}$ is a unique overtaking optimal program with the initial state $x_{0}$.

\section{REFERENCES}

[1] S. M. Aseev, M. I. Krastanov, V. M. Veliov, Optimality conditions for discrete-time optimal control on infinite horizon, Pure Appl. Funct. Anal. 2 (2017), 395-409.

[2] S. Aubry, P. Y. Le Daeron, The discrete Frenkel-Kontorova model and its extensions I, Physica D 8 (1983), 381-422.

[3] M. Bachir, J. Blot, Infinite dimensional infinite-horizon Pontryagin principles for discrete-time problems, Set-Valued Var. Anal. 23 (2015), 43-54.

[4] M. Bachir, J. Blot, Infinite dimensional multipliers and Pontryagin principles for discrete-time problems, Pure Appl. Funct. Anal. 2 (2017) , 411-426.

[5] J. Baumeister, A. Leitao, G. N. Silva, On the value function for nonautonomous optimal control problems with infinite horizon, Systems Control Lett. 56 (2007), 188-196.

[6] J. Blot, P. Cartigny, Optimality in infinite-horizon variational problems under sign conditions, J. Optim. Theory Appl. 106 (2000), 411-419.

[7] J. Blot, N. Hayek, Sufficient conditions for infinite-horizon calculus of variations problems, ESAIM Control Optim. Calc. Var. 5 (2000), 279-292.

[8] J. Blot, N. Hayek, Infinite-horizon Optimal Control in the Discrete-time Framework, SpringerBriefs in Optimization, New York, 2014.

[9] D. A. Carlson, A. Haurie, A Leizarowitz, Infinite Horizon Optimal Control, Springer-Verlag, Berlin, 1991.

[10] T. Damm, L. Grune, M. Stieler, K. Worthmann, An exponential turnpike theorem for dissipative discrete time optimal control problems, SIAM J. Control Optim. 52 (2014), 1935-1957.

[11] V. Gaitsgory, L. Grune, N. Thatcher, Stabilization with discounted optimal control, Sys. Control Lett. 82 (2015), 91-98.

[12] V. Gaitsgory, M. Mammadov, L. Manic, On stability under perturbations of long-run average optimal control problems, Pure Appl. Funct. Anal. 2 (2017), 461-476.

[13] D. Gale, On optimal development in a multi-sector economy, Rev. Econ. Stud. 34 (1967), 1-18.

[14] M. Gugat, E. Trelat, E. Zuazua, Optimal Neumann control for the 1D wave equation: finite horizon, infinite horizon, boundary tracking terms and the turnpike property, Sys. Control Lett. 90 (2016), 61-70.

[15] P. J. Hammond, Consistent Planning and Intertemporal Welfare Economics, University of Cambridge, Cambridge, 1974.

[16] P. J. Hammond, Agreeable plans with many capital goods, Rev. Econ. Stud. 42 (1975), 1-14.

[17] P. J. Hammond, J. A. Mirrlees, Agreeable Plans, Models of Economic Growth, pp. 283-299, Wiley, New York, 1973.

[18] N. Hayek, Infinite horizon multiobjective optimal control problems in the discrete time case, Optimization 60 (2011), 509-529.

[19] M. Ali Khan, T. Mitra, On choice of technique in the Robinson-Solow-Srinivasan model, Int. J. Econ. Theory 1 (2005), 83-110.

[20] M. Ali Khan, T. Mitra, Undiscounted optimal growth in the two-sector Robinson-Solow-Srinivasan model: a synthesis of the value-loss approach and dynamic programming, Econ. Theory 29 (2006), 341-362.

[21] M. Ali Khan, T. Mitra, Discounted optimal growth in the two-sector RSS model: a geometric investigation, Adv. Math. Econ. 8 (2006) 349-381. 
[22] M. Ali Khan, T. Mitra, Optimal growth in a two-sector RSS model without discounting: a geometric investigation, Jpn. Econ. Rev. 58 (2007) 191-225.

[23] M. Ali Khan, T. Mitra, Optimal growth under discounting in the two-sector Robinson-Solow-Srinivasan model: a dynamic programming approach, J. Difference Equ. Appl. 13 (2007), 151-168.

[24] M. Ali Khan, T. Mitra, Growth in the Robinson-Solow-Srinivasan model: undiscounted optimal policy with a strictly concave welfare function, J. Math. Econom. 44 (2008), 707-732.

[25] M. Ali Khan, T. Mitra, Impatience and dynamic optimal behavior: a bifurcation analysis of the RobinsonSolow-Srinivasan model, Nonlinear Anal. 75 (2012), 1400-1418.

[26] M. Ali Khan, T. Mitra, Discounted optimal growth in a two-sector RSS model: a further geometric investigation, Adv. Math. Econ. 17 (2013), 39-70.

[27] M. Ali Khan, A. Piazza, On the non-existence of optimal programs in the Robinson-Solow-Srinivasan (RSS) model, Econ. Lett. 109 (2010), 94-98.

[28] M. Ali Khan, A. Piazza, The concavity assumption on felicities and asymptotic dynamics in the RSS model, Set-Valued Var. Anal. 19 (2011), 135 ?156.

[29] M. Ali Khan, A. Piazza, An overview of turnpike theory: towards the discounted deterministic case, Adv. Math. Econ. 14 (2011), 39-67.

[30] M. Ali Khan, A. J. Zaslavski, On a uniform turnpike of the third kind in the Robinson-Solow-Srinivasan model, J. Economics 92 (2007), 137-166.

[31] M. Ali Khan, A. J. Zaslavski, On existence of optimal programs: the RSS model without concavity assumptions on felicities, J. Math. Economics 45 (2009), 624-633.

[32] M. Ali Khan, A. J. Zaslavski, On two classical turnpike results for the Robinson-Solow-Srinivisan (RSS) model, Adv. Math. Econom. 13 (2010), 47-97.

[33] M. Ali Khan, A. J. Zaslavski, On locally optimal programs in the RSS (Robinson-Solow-Srinivasan) model, J. Economics 99 (2010), 65-92.

[34] A. Leizarowitz, Infinite horizon autonomous systems with unbounded cost, Appl. Math. Optim. 13 (1985) $19-43$.

[35] A. Leizarowitz, V. J. Mizel, One dimensional infinite horizon variational problems arising in continuum mechanics, Arch. Rational Mech. Anal. 106 (1989), 161-194.

[36] V. Lykina, S. Pickenhain, M. Wagner, Different interpretations of the improper integral objective in an infinite horizon control problem, J. Math. Anal. Appl. 340 (2008), 498-510.

[37] V. L. Makarov, A. M. Rubinov, Mathematical Theory of Economic Dynamics and Equilibria, Springer-Verlag, New York, 1977.

[38] M. Mammadov, Turnpike theorem for an infinite horizon optimal control problem with time delay, SIAM J. Control Optim. 52 (2014), 420-438.

[39] M. Marcus, A. J. Zaslavski, The structure of extremals of a class of second order variational problems, Ann. Inst. H. Poincare, Anal. Non Lineare 16 (1999), 593-629.

[40] L. W. McKenzie, Turnpike theory, Econometrica 44 (1976), 841-866.

[41] B. S. Mordukhovich, Optimal control and feedback design of state-constrained parabolic systems in uncertainly conditions, Appl. Anal. 90 (2011), 1075-1109.

[42] B. S. Mordukhovich, I. Shvartsman, Optimization and feedback control of constrained parabolic systems under uncertain perturbations, Optimal Control, Stabilization and Nonsmooth Analysis, Lecture Notes Control Inform. Sci. pp. 121-132, Springer, 2004.

[43] N. Okishio, Technical choice under full employment in a socialist economy, Economic Journal 76 (2011), $585-592$.

[44] S. Pickenhain, V. Lykina, M. Wagner, On the lower semicontinuity of functionals involving Lebesgue or improper Riemann integrals in infinite horizon optimal control problems, Control Cybernet. 37 (2008), 451468.

[45] A. Porretta, E. Zuazua, Long time versus steady state optimal control, SIAM J. Control Optim. 51 (2013), 4242-4273.

[46] T. Prieto-Rumeau, O. Hernandez-Lerma, Bias and overtaking equilibria for zero-sum continuous-time Markov games, Math. Methods Oper. Res. 61 (2005), 437-454. 
[47] R. Radner, Paths of economics growth that are optimal with regard only to final states: a turnpike theorem, Rev. Economic Stud. 28 (1961), 98-104.

[48] J. Robinson, Exercises in Eeconomic Analysis, MacMillan, London, 1960.

[49] J. Robinson, A model for accumulation proposed by J.E. Stiglitz, Economic Journal 79 (1969), 412-413.

[50] N. Sagara, Recursive variational problems in nonreflexive Banach spaces with an infinite horizon: an existence result, Discrete Contin. Dyn. Syst. Ser. S 11 (2018), 1219-1232.

[51] P. A. Samuelson, A catenary turnpike theorem involving consumption and the golden rule, Amer. Economic Rev. 55 (1965), 486-496.

[52] R. M. Solow, Substitution and fixed proportions in the theory of capital, Rev. Economic Stud. 29 (1962), 207-218.

[53] T. N. Srinivasan, Investment criteria and choice of techniques of production, Yale Economic Essays 1 (1962), $58-115$.

[54] J. E. Stiglitz, A note on technical choice under full employment in a socialist economy, Economic Journal 78 (1968), 603-609.

[55] J. E. Stiglitz, Reply to Mrs. Robinson on the choice of technique, Economic Journal 80 (1970), 420-422.

[56] J. E. Stiglitz, Recurrence of Techniques in a Dynamic Economy, Models of economic growth (J. Mirrlees and N. H. Stern, eds.), Wiley, New York, 283?299, 1973.

[57] E. Trelat, C. Zhang, E. Zuazua, Optimal shape design for 2D heat equations in large time, Pure Appl. Funct. Anal. 3 (2018), 255-269.

[58] C. C. von Weizsacker, Existence of optimal programs of accumulation for an infinite horizon, Rev. Econ. Studies 32 (1965), 85-104.

[59] A. J. Zaslavski, Optimal programs in the RSS model, Int. J. Economic Theory 1 (2005), 151-165.

[60] A. J. Zaslavski, Good programs in the RSS model with a nonconcave utility function, J. Ind. Management Optim. 2 (2006), 399-423.

[61] A. J. Zaslavski, Turnpike Properties in the Calculus of Variations and Optimal Control, Springer, New York, 2006.

[62] A. J. Zaslavski, The structure of good programs in the RSS Model, Proceedings of the Annual Meeting at the Kyoto University 1654 (2009), 166-178,

[63] A. J. Zaslavski, Good solutions for a class of infinite horizon discrete-time optimal control problems, Taiwanese J. Math. 13 (2009), 1637-1669.

[64] A. J. Zaslavski, Turnpike results for a class of infinite horizon discrete-time optimal control problems arising in economic dynamics, Set-Valued Var. Anal. 17 (2009), 285-318.

[65] A. J. Zaslavski, Existence and structure of solutions for an infinite horizon optimal control problem arising in economic dynamics, Adv. Differential Equ. 14 (2009), 477-496.

[66] A. J. Zaslavski, Good locally maximal programs for the Robinson-Solow-Srinivasan (RSS) model, Adv. Math. Economics 13 (2010), 161-176.

[67] A. J. Zaslavski, Overtaking optimal solutions for a class of infinite horizon discrete-time optimal control problems, Dynamics of Continuous, Discrete and Impulsive Systems, Ser. B 17 (2010), 607-620.

[68] A. J. Zaslavski, Locally maximal solutions of control systems arising in economic dynamics, Commun. Appl. Nonlinear Anal. 17 (2010), 61-68.

[69] A. J. Zaslavski, Two turnpike results for a continuous-time optimal control systems, Proceedings of an International Conference, Complex Analysis and Dynamical Systems IV: function theory and optimization 553 (2011), 305-317.

[70] A. J. Zaslavski, Stability of a turnpike phenomenon for the Robinson-Solow-Srinivasan model, Dyn. Sys. Appl. 20 (2011), 25-44.

[71] A. J. Zaslavski, A turnpike property of approximate solutions of an optimal control problem arising in economic dynamics, Dyn. Sys. Appl. 20 (2011), 395-422.

[72] A. J Zaslavski, Weakly agreeable programs for the Robinson-Solow-Srinivasan (RSS) model, Optimization theory and related topics, Contemp. Math. 568 (2012), 259-271.

[73] A. J. Zaslavski, On turnpike properties of approximate optimal programs of the discrete-time RobinsonSolow-Srinivasan model, Commun. Appl. Anal. 17 (2013), 129-145. 
[74] A. J. Zaslavski, On a class of discrete-time optimal control problems arising in economic dynamics, PanAmer. Math. J. 23 (2013), 1-12.

[75] A. J. Zaslavski, Stability of the turnpike phenomenon for a convex optimal control problem arising in economic dynamics, Commun. Appl. Anal. 17 (2013), 271-288.

[76] A. J. Zaslavski, Turnpike Phenomenon and Infinite Horizon Optimal Control, Springer Optimization and Its Applications, New York, 2014.

[77] A. J. Zaslavski, Structure of solutions of optimal control problems on large intervals: a survey of recent results, Pure Applied Funct. Anal. 1 (2016), 123-158.

[78] A. J. Zaslavski, Discrete-time Optimal Control and Games on Large Intervals, Springer Optimization and Its Applications, Springer, Cham, 2017.

[79] A. J. Zaslavski, Equivalence of optimality criterions for discrete time optimal control problems, Pure Appl. Funct. Anal. 3 (2018), 505-517.

[80] A. J. Zaslavski, Turnpike Conditions in Infinite Dimensional Optimal Control, Springer Optimization and Its Applications, Springer, Cham, 2019.

[81] A. J. Zaslavski, Optimal Control Problems Arising in the Forest Management, Springer Briefs in Optimization, Springer, Cham, 2019. 